\title{
Satisfacción y fuentes de presión laboral en docentes universitarios de Lima metropolitana
}

David Álvarez Flores

El presente trabajo, de tipo correlacional, analizó la relación entre las Fuentes de Presión Laboral y la Satisfacción Laboral de los docentes universitarios de Lima metropolitana. Buscó conocer los factores que tienen mayor incidencia en la Satisfacción Laboral y las Fuentes de Presión Laboral en esta población. Se utilizó un diseño de tipo transeccional correlacional. La población considerada es el total de docentes universitarios de Lima metropolitana. La muestra está conformada por 506 docentes seleccionados por conglomerados. Los instrumentos utilizados fueron la Escala de Satisfacción Docente y el Inventario de presiones a las que se enfrenta el docente. Los instrumentos presentaron validez de constructo y confiabilidad por consistencia interna. Se concluyó que las Fuentes de Presión Laboral y la Satisfacción Laboral se relacionan de manera inversa.

Satisfacción Laboral / Fuentes de Presión Laboral / estrés / docencia universitaria

Job satisfaction and sources of work pressure on college professors in Metropolitan Lima

This correlational research sought to investigate the relationship between the sources of work pressure and job satisfaction among university professors in metropolitan Lima. In this study it was used a cross-sectional correlational design. The sample consisted of 506 university professors selected by clusters. The following instruments were used: Personal Data Questionnaire, Teaching Satisfaction Scale using a Lickert-type scale, and an Inventory of Teaching Work pressure. The instruments showed construct validity and internal consistency reliability. The findings show that sources of work pressure and job satisfaction are inversely related.

job satisfaction / sources of work pressure / stress / university teaching 
El estudio de la educación universitaria peruana presenta ciertas dificultades. Se señalan la escasez de investigaciones, la dispersión de los pocos estudios realizados y la confiabilidad de la información existente. Las investigaciones sobre el docente universitario peruano son escasas; la mayoría de los trabajos realizados con este importante sector se han orientado a una recopilación de datos, como insumo para posteriores análisis. Otra de las dificultades en la comprensión de la educación superior en el país radica en la dispersión de los pocos datos estadísticos existentes y en el poco esfuerzo de recolección sistemática y la integración de estos. Presentan un conjunto de cifras estadísticas referidas a la demanda y la oferta de la educación, a la situación del cuerpo docente, al financiamiento y eficiencia del sistema de la educación superior $\mathrm{y}$, finalmente, al desarrollo de la investigación científica y tecnológica (Grade, 1990). Un tercer aspecto es la falta de información confiable, especialmente la económica. Una de las razones es la reserva con que se tratan estos temas en las universidades privadas, indicio de la carencia de normas reguladoras por parte del Estado (Sota, 2000).

Nuestras universidades difieren en calidad académica, tamaño, perfil profesional e infraestructura, de tal manera que la categoría "educación superior peruana" solo tiene sentido cuando se refiere a una heterogeneidad extrema.
En ese contexto, el papel de las universidades cobra en la actualidad mayor trascendencia, en razón de que se percibe una creciente necesidad de entrenamiento para mayores y distintas habilidades en las economías más desarrolladas. Una primera dimensión se refiere a que en las economías dirigidas por el conocimiento, los trabajadores requieren un mayor nivel de habilidades. La segunda dimensión está referida a la creciente importancia de la educación continua, por la necesidad de actualización constante en conocimiento y habilidades; en este caso se espera que la futura población universitaria no esté constituida solo por egresados de secundaria, sino por una clientela muy diversa. La tercera dimensión la constituye el atractivo de los títulos universitarios con aplicación internacional (Salmi, 2000).

Algunos estudios reportan datos importantes para este análisis. Así, las cifras sobre la distribución profesor/alumno en el año 1983 en el Perú indican que se encontraban alrededor de 17.42, a diferencia de otros países como Argentina (10.35), Brasil (12.06), Cuba (12.14) y México (10.81). Asimismo, la distribución porcentual del tiempo de los docentes de universidades públicas según las funciones realizadas indican que en el año 1974, el 44,5\% se dedica al dictado de clases, el $12 \%$ a investigación, el $11,1 \%$ a gobierno, el $6 \%$ se dedica a la preparación de clases, el 5,9\% a conse- 
jería, el 1,2\% a prácticas profesionales, el $2,1 \%$ a producción de bienes y servicios, el $2,7 \%$ a capacitación, el $2,9 \%$ a proyección social y $11,32 \%$ a otros (Grade, 1990).

En 1996, el Primer Censo Nacional Universitario se propuso como objetivo conocer la realidad universitaria, en aspectos organizacionales y de infraestructura física, académica, económica, administrativa y equipamiento científico tecnológico; asimismo, indagar sobre las características demográficas, socioeconómicas y académicas del personal docente, trabajadores no docentes y estudiantes. Algunos de sus resultados señalan que, en relación con los docentes, el tiempo promedio de servicios es 9,1 años; el número de horas promedio de dictado de clases asciende a 15,8 y el promedio de asignaturas a su cargo es 2,3 . Con relación a la situación laboral, el $35 \%$ de los docentes es contratado y el $53 \%$ de los profesores de Lima metropolitana trabaja en actividades complementarias a la docencia. En cuanto al acceso a la información el 19\% tiene acceso a internet y el $38 \%$ tiene acceso a computadora propia. En cuanto a la percepción de desarrollo de los docentes, el 20\% considera que esta es excelente y el $59 \%$ que es buena. Considerando la infraestructura física, el $9 \%$ la percibe como excelente y el $49 \%$ como buena. En relación con la infraestructura académica, el $5,5 \%$ la considera excelente y el $36,3 \%$, buena (INEI, 2006).
Asimismo, es importante conocer la percepción sobre algunos aspectos de la situación de las universidades, tales como el reconocimiento del docente y la confianza en esa institución. El rol del docente universitario tiene poco reconocimiento en la opinión pública de Lima metropolitana; así, el 24,7\% considera que el aspecto más importante de la universidad es el nivel académico, el 19,2\% el acceso a becas, el $17,9 \%$ la investigación, el 2,2\% el profesor. Asimismo, se considera que el principal problema que enfrenta la universidad es el excesivo número de instituciones $(15,9 \%)$, pensiones $(15,4 \%)$, infraestructura $(12,6 \%)$, tecnología $(12,6 \%)$, plan de estudios $(12,3 \%)$ y el docente $(6,5 \%)$. El nivel de confianza de las universidades públicas llega a $74 \%$, en tanto que el de las privadas llega a $79 \%$. En cuanto a la calidad de estas, el $43 \%$ sostiene que la universidad pública es muy buena/buena y la privada llega a $71 \%$ en esa misma categoría (Universidad de Lima, 2004).

Las condiciones de trabajo del docente universitario varían de una institución a otra en función de la disponibilidad presupuestal, la legislación vigente, las condiciones políticas del país, la filosofía de la propia universidad, entre otros factores. Del mismo modo, los profesores se enfrentan en su desempeño a un conjunto de factores, como la interacción docente-alumno, los estilos de administración de la universidad, el número de alumnos por sec- 
ción, la relación entre horas lectivas y no lectivas, la metodología, evaluación del profesor a cargo de los alumnos y los estamentos administrativos, para citar algunos. Precisando aún más las condiciones de trabajo, podemos incluir otras, como creatividad, responsabilidad, independencia, variedad, utilización al máximo de su preparación académica o experiencia profesional, el sentimiento de realización, las buenas relaciones interpersonales o el reconocimiento que logre por sus actividades docentes. Los responsables de la administración de las universidades deben procurar mantener estas condiciones de tal forma que constituyan un factor de atracción de los docentes. En la medida en que se proporcionen estas condiciones pueden proveer algún grado de satisfacción o constituirse en una fuente de presión laboral en la labor académica que desempeña.

En la actualidad, no se cuenta con estudios realizados en nuestro país que permitan visualizar la forma en que estas condiciones de trabajo son administradas por las autoridades universitarias, ni en qué medida los docentes perciben que tales condiciones constituyen Fuentes de Presión Laboral o les brinde algún nivel de Satisfacción Laboral.

El desconocimiento de estos aspectos del docente universitario puede ocasionar que las políticas emanadas de las autoridades universitarias no logren los efectos deseados para alcanzar la calidad de la enseñanza, la integración e identificación del docente con la universidad y su bienestar.

La Satisfacción Laboral es una consecuencia del desempeño de una tarea, empleo o función. Asimismo, es una respuesta que experimenta el individuo, tanto hacia el trabajo como hacia cada uno de los aspectos específicos o generales que lo determinan. Las fuentes o factores del trabajo relevantes que permiten que el trabajador se sienta satisfecho laboralmente son la satisfacción con el trabajo mismo, el salario, las promociones, el reconocimiento, los beneficios, condiciones de trabajo, la supervisión, con los compañeros y con la compañía y la división (Herzberg, 1959; Dawis Lofquist y Weis, 1968; Locke, 1976, en en Peiró, 1966)).

Asimismo, frecuentemente se trata de relacionar la satisfacción laboral con la conducta organizacional, donde se deben considerar la productividad, el ausentismo y la rotación de personal. La revisión de investigaciones arroja poca evidencia sobre la existencia de una relación simple o apreciable entre la satisfacción de los trabajadores y su nivel de ejecución. Igualmente, se ha reportado una correlación negativa significativa entre satisfacción y ausentismo (Bayfield \& Crockett, 1955). También se ha encontrado que en la decisión de abandonar una organización no solo interviene el nivel de satisfacción de las expectativas y necesida- 
des del trabajador sino también las expectativas de un mejor puesto en la organización. (March \& Simons, 1977, en Peiró, 1987).

No se conoce cómo estos aspectos, facetas o factores del ambiente educacional pueden estar incidiendo en la satisfacción laboral de los docentes, teniendo en cuenta su condición (nombrado o contratado), categoría (principal, asociado, auxiliar), dedicación (tiempo parcial o completo), género (masculino o femenino), la facultad a la que se encuentra adscrito y el tipo de gestión (pública o privada). Igualmente, se desconoce cuáles son los factores que pueden estar contribuyendo a incrementar el estrés laboral en los docentes.

En el Perú no se han realizado otros estudios sobre satisfacción laboral en docentes universitarios, en cambio en otros países sí existen tales investigaciones en esta población, pero se presentan dificultades en la comparación de ciertos aspectos en razón del marco teórico empleado, instrumentos de medición, diversidad de factores, metodología y variables consideradas (Reimel, 1994; Polanco, 1994; Marchand \& Pérez de Maldonado, 1997).

El estudio del estrés ha cobrado en la actualidad gran interés, especialmente para la organización, que para remediar esta dolencia debe tratar de identificar a los trabajadores con mayores posibilidades de sufrirla. Se señala que los diez puestos de trabajo cuyos ocu- pantes tienen una alta probabilidad de "quemarse" son: controlador de tráfico aéreo, comprador de la industria de la confección, ejecutivo de seguros, abogado, enfermera, médico, psicólogo, asistente social, agente de bolsa, profesor $\mathrm{y}$, por último, el conductor de camiones (Batten, 1985, citado por Byars \& Rue, 1996).

El interés por el estrés en el personal docente se ha centrado en dos aspectos: las consecuencias de estar sometido a una fuente continua de estrés, angustia e insatisfacción, conocida como desgaste profesional, burnout, quemazón laboral o desgaste laboral. La otra faceta de interés ha sido la de conocer las fuentes de presión que afectan a esta importante población.

En nuestro país se han realizado estudios sobre estrés en docentes de Lima metropolitana en los niveles primario, secundario y universitario. Las fuentes de presión laboral a las que se enfrenta el docente de educación primaria son las provenientes de la labor educativa en el aula, la vida cotidiana en el aula, la organización administrativa y la flexibilidad de la tarea docente (Fernández, 2002). Debe precisarse que estas fuentes de presión laboral serán asumidas en el presente estudio. En docentes de educación secundaria se analizó el síndrome del burnout considerando las variables sexo, tipo de colegio y tiempo de servicios (Delgado, Escurra \& Atalaya, 2003). En el sector de docentes universitarios 
se estudió la posible presencia del síndrome del "quemado" por estrés laboral asistencial, en una muestra de docentes de la Universidad Nacional Mayor de San Marcos de diferentes áreas; considerando como variables el área de especialidad, sexo, estado civil, práctica de deporte, problemas de salud y condición docente (Ponce et al., 2005).

En otras realidades se encuentran las investigaciones relacionadas con el tema y la población de estudio (Guerrero, 2003; Reimel, 1994; Polanco, 1994; Marchand \& Pérez Maldonado,1997; González,1988; Galaz, 2002; U. S. Department of Education, National Center for Education Statistics, 1999, en NEA, 2000).

El conocimiento de las situaciones $o$ factores que hacen que el docente universitario se sienta satisfecho o no y con algún nivel de presión de algunas de las condiciones que le brindan las instituciones educativas es de vital importancia por las repercusiones que acarrean a las universidades. Los factores o situaciones de insatisfacción o presión laboral pueden afectar a las condiciones académicas, administrativas y personales del docente. En el aspecto académico, puede redundar en la transmisión de conocimientos, metodología, evaluación, obsolescencia de conocimientos, falta de actualización académica, entre otros. Las consecuencias administrativas pueden reflejarse en ausentismo, tardanzas, faltas disci- plinarias, deshonestidad, renuncia y despidos, entre otros. En el aspecto personal puede redundar en el deterioro de su autoestima, depresión u otros trastornos emocionales y somáticos.

La presente investigación está orientada a encontrar respuesta a la siguiente interrogante: ¿Qué relación existe entre las fuentes de presión laboral y la satisfacción laboral en los docentes universitarios de Lima metropolitana?

\section{HIPÓTESIS DE TRABAJO}

De acuerdo con el análisis bibliográfico efectuado, se establecieron las siguientes hipótesis de trabajo:

- Existe relación inversa entre Presión Laboral y Satisfacción Laboral de los docentes universitarios de Lima metropolitana.

- La Satisfacción Laboral de los docentes universitarios de las universidades estatales difiere de la Satisfacción Laboral de los docentes de las universidades privadas de Lima metropolitana.

- Las Fuentes de Presión Laboral de los docentes de universidades estatales difiere de las Fuentes de Presión Laboral de los docentes de universidades privadas de Lima metropolitana.

- Los factores de las Fuentes de Presión Laboral de los docentes influyen sobre los factores de la Satisfacción Laboral. 
- Existen diferencias en la Satisfacción Laboral y en los factores de las Fuentes de Presión Laboral, según las variables asignadas: condición de nombrado o de contratado, dedicación, categoría, labores en una o más universidades y formación en universidad estatal o privada.

- Existen diferencias en la Satisfacción Laboral y en los factores de las Fuentes de Presión Laboral, según las variables demográficas: estado civil, género y lugar de nacimiento.

\section{MÉTOdO DE INVESTIGACIÓN}

El estudio corresponde a una investigación de tipo sustantiva, en razón de que trata de responder a un problema teórico y se orienta a describir la realidad del docente universitario en cuanto a la satisfacción laboral y las fuentes de presión laboral.

La investigación es correlacional y comparativa (Alarcón, 1991; Hernández, Fernández \& Baptista, 2006), debido a que permite recoger evidencias de hechos, concretándose en tomar medidas acerca de la Satisfacción Laboral y las Fuentes de Presión, e indagar por supuestas relaciones entre ellas y efectuar sus contrastaciones.

\section{DISEÑO DE INVESTIGACIÓN}

El diseño de investigación es transeccional, correlacional y comparativo (Hernández, Fernández \& Baptista,
2006; Sánchez \& Reyes, 2002) y se realiza en docentes de universidades estatales y privadas de Lima metropolitana.

\section{Población y muestra de estudio}

La población considerada en la presente investigación estuvo conformada por 19.707 docentes de las universidades estatales y privadas de Lima metropolitana, tomando como fuente a la Asamblea Nacional de Rectores (2005). Esta cifra representa el 45,46\% de la población docente nacional; los docentes de esta circunscripción representan el $32,78 \%$ y el $58,43 \%$ de los docentes de universidades estatales y privadas del país, respectivamente.

La muestra estuvo integrada por 506 docentes de diez universidades, cuatro estatales y seis privadas, guardando la proporción con la población de cada una de ellas. El tamaño de la muestra se calculó de acuerdo con la fórmula de Cochrane para poblaciones inferiores a los cien mil casos, estableciéndose como parámetros un nivel de ocurrencia del $50 \%$, un margen de error del 5\% y un nivel de confianza bilateral del $95 \%(z=1.96)$.

La selección de la muestra se realizó considerando el muestreo probabilístico polietápico por conglomerados (Alarcón, 1991). La primera etapa (unidad primaria de muestreo) consistió en seleccionar a las universidades que participaron en la muestra. Se con- 
sideró como criterio de inclusión a las cuatro universidades públicas y la mitad de las privadas de Lima metropolitana que contaran como mínimo con cien docentes. De esta manera, fueron consideradas las siguientes:

- Universidades estatales: Universidad Nacional Mayor de San Marcos, Universidad Nacional de Ingeniería, Universidad Nacional Agraria La Molina, Universidad Nacional Federico Villarreal.

- Universidades privadas: Pontificia Universidad Católica del Perú, Uni- versidad Particular San Martín de Porres, Universidad de Lima, Universidad Particular Inca Garcilaso de la Vega, Universidad Particular Ricardo Palma, Universidad San Ignacio de Loyola.

Posteriormente, se realizó la inclusión de las facultades de cada universidad (unidad secundaria de muestreo), teniendo como criterio considerar el $50 \%$ del número de facultades. De esta manera, las facultades consideradas para cada universidad fueron las siguientes (tablas 1 y 2 ):

Tabla 1

Facultades seleccionadas entre las universidades estatales

\begin{tabular}{lccl}
\hline \multicolumn{1}{c}{ Universidades } & $\begin{array}{c}\text { Facultades } \\
\text { número }\end{array}$ & $\begin{array}{c}\text { Total } \\
\text { muestra }\end{array}$ & Facultades \\
\hline $\begin{array}{l}\text { Universidad Nacional } \\
\text { Mayor de San Marcos }\end{array}$ & 20 & 10 & $\begin{array}{l}\text { Letras y Ciencias Humanas, Farmacia y Bioquímica, } \\
\text { Ingeniería Industrial, Medicina Veterinaria, Ciencias } \\
\text { Económicas, Psicología, Ingeniería de Sistemas e } \\
\text { Informática, Ciencias Matemáticas, Ciencias Sociales } \\
\text { y Ciencias Biológicas }\end{array}$ \\
$\begin{array}{lccl}\text { Universidad Nacional } \\
\text { Federico Villarreal }\end{array}$ & 18 & 9 & $\begin{array}{l}\text { Ingeniería Geográfica y Ambiental, Psicología, } \\
\text { Ciencias Sociales, Arquitectura y Urbanismo, }\end{array}$ \\
$\begin{array}{lll}\text { Universidad Nacional } \\
\text { de Ingeniería }\end{array}$ & 11 & 6 & $\begin{array}{l}\text { Administración, Humanidades, Electrónica e } \\
\text { Informática, Ciencias Económicas e Ingeniería Civil. } \\
\text { Ingeniería Eléctrica y Electrónica, Ingeniería } \\
\text { Mecánica, Ciencias, Ingeniería de Petróleo y } \\
\text { Petroquímica, Arquitectura, Urbanismo y Arte e } \\
\text { Ingeniería Económica y Ciencias Sociales. }\end{array}$ \\
$\begin{array}{l}\text { Universidad Nacional } \\
\text { Agraria La Molina }\end{array}$ & 9 & 4 & $\begin{array}{l}\text { Ingeniería Agrícola, Ciencias, Agronomía, Industrias } \\
\text { Alimentarias y Pesquería. }\end{array}$ \\
\hline
\end{tabular}


Tabla 2

Facultades seleccionadas entre las universidades privadas

\begin{tabular}{|c|c|c|c|}
\hline Universidades & $\begin{array}{l}\text { Facultades } \\
\text { número }\end{array}$ & $\begin{array}{c}\text { Total } \\
\text { muestra }\end{array}$ & Facultades \\
\hline $\begin{array}{l}\text { Pontificia Universidad } \\
\text { Católica del Perú }\end{array}$ & 9 & 5 & $\begin{array}{l}\text { Ciencias y Artes de la Comunicación, Letras y } \\
\text { Ciencias Humanas, Arquitectura y Urbanismo, } \\
\text { Administración y Contabilidad y Ciencias Sociales. }\end{array}$ \\
\hline $\begin{array}{l}\text { Universidad San Martín } \\
\text { de Porres }\end{array}$ & 9 & 5 & $\begin{array}{l}\text { Ingeniería y Arquitectura, Odontología, } \\
\text { Comunicación, Turismo y Psicología, Ciencias } \\
\text { Administrativas y Relaciones Industriales y } \\
\text { Educación. }\end{array}$ \\
\hline Universidad de Lima & 8 & 5 & $\begin{array}{l}\text { Comunicación, Contabilidad, Administración, } \\
\text { Ingeniería de Sistemas e Ingeniería Industrial }\end{array}$ \\
\hline $\begin{array}{l}\text { Universidad Garcilaso } \\
\text { de la Vega }\end{array}$ & 11 & 6 & $\begin{array}{l}\text { Educación, Ingeniería Administrativa e Industrial, } \\
\text { Ciencias Contables, Ingeniería de Sistemas y } \\
\text { Cómputo, Ciencias Administrativas y Ciencias } \\
\text { Económicas }\end{array}$ \\
\hline $\begin{array}{l}\text { Universidad Ricardo } \\
\text { Palma }\end{array}$ & 7 & 5 & $\begin{array}{l}\text { Medicina Humana, Ciencias Biológicas, Arquitectura } \\
\text { y Urbanismo, Lenguas Modernas y Psicología }\end{array}$ \\
\hline $\begin{array}{l}\text { Universidad San } \\
\text { Ignacio de Loyola }\end{array}$ & 8 & 5 & $\begin{array}{l}\text { Economía, Marketing, Administración Hotelera } \\
\text { Administración y Ciencias y Tecnología de la } \\
\text { Comunicación. }\end{array}$ \\
\hline
\end{tabular}

Finalmente, se determinó la muestra por cada una de las universidades incluidas en el estudio, quedando conformada de la siguiente manera (tabla 3):

Tabla 3

Determinación final de la muestra por universidades

\begin{tabular}{lccc}
\hline \multicolumn{1}{c}{ Universidad } & $\begin{array}{c}\text { Población } \\
\text { de docentes }\end{array}$ & $\begin{array}{c}\text { Muestra por } \\
\text { universidad }\end{array}$ & $\%$ \\
\hline Universidad Nacional Mayor de San Marcos & 2.948 & 100 & 19,8 \\
Universidad Nacional de Ingeniería & 1.328 & 35 & 6,9 \\
Universidad Nacional Agraria Agraria La Molina & 484 & 17 & 3,4 \\
Universidad Nacional Federico Villarreal & 2.089 & 60 & 11,9 \\
Pontificia Universidad Católica del Perú & 1.889 & 78 & 15,4 \\
Universidad de San Martín de Porres & 2.156 & 80 & 15,8 \\
Universidad de Lima & 845 & 37 & 7,3 \\
Universidad Garcilaso de la Vega & 992 & 54 & 10,7 \\
Universidad Ricardo Palma & 750 & 30 & 5,9 \\
Universidad San Ignacio de Loyola & 359 & 15 & 3,0 \\
Total & 13.840 & 506 & 100,0 \\
\hline
\end{tabular}


COMPOSICIÓN Y DESCRIPCIÓN DE LA MUESTRA

La muestra está conformada por 212 $(42 \%)$ docentes universitarios provenientes de universidades estatales y 294 $(58 \%)$ de universidades particulares. Las edades se encuentran entre los 25 a 75 años; el estado civil corresponde a $39 \%$ de solteros y $49 \%$ de casados; en cuanto al género $74 \%$ son varones y $26 \%$ mujeres; según lugar de nacimiento, $64 \%$ provienen de Lima y $35 \%$ de provincias. En relación con el aspecto académico, $95 \%$ enseña en pregrado y $5 \%$ en posgrado; en la condición de nombrado se encuentra el $62 \%$ y como contratado el $38 \%$; según la dedicación, el $37 \%$ trabaja a tiempo completo, $47 \%$ a tiempo parcial y $15 \%$ a dedicación exclusiva; el 21 por ciento pertenece a la categoría principal, el $38 \%$ a la de asociado y $38 \%$ es auxiliar; el $39 \%$ trabaja sólo en esa universidad y el 59\% trabaja en más de una universidad.

\section{INSTRUMENTOS}

Los instrumentos utilizados en la presente investigación fueron: el cuestionario de datos personales, la escala de satisfacción docente y el inventario de presiones a las que se enfrenta el docente.

\section{Cuestionario de datos personales}

Este cuestionario busca obtener información sobre las características del docente. Consta de dos secciones: la primera indaga acerca de los datos sociodemográficos del docente; la segunda está referida a su condición laboral en la universidad.

\section{Cuestionario sobre satisfacción docente}

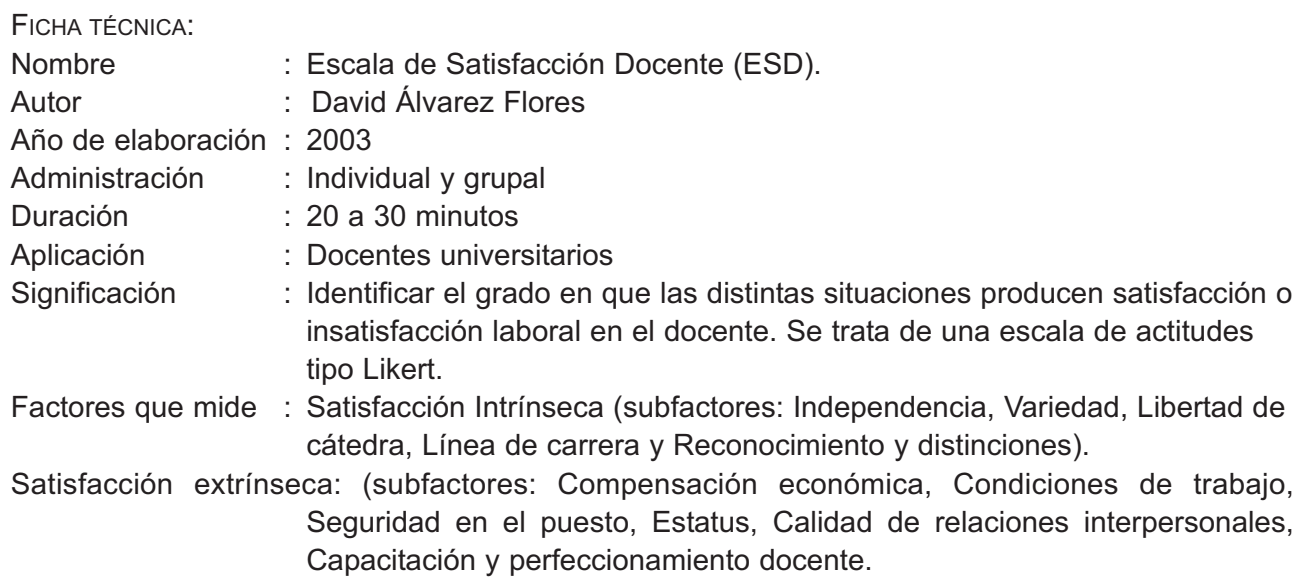




\section{Diseño de LA PRUEBA}

En primer lugar, se elaboró la tabla de especificaciones, que contiene las variables, la definición operacional y los respectivos indicadores; luego, de acuerdo con los factores establecidos, se elaboraron entre cinco y seis ítems por cada uno de ellos; y, finalmente, la versión final de todos los ítems creados fue sometida a un proceso de validez de contenido utilizando el criterio de jueces. Para este fin, a un grupo de diez jueces conformados por psicólogos especialistas en el tema se les presentó la versión final de la prueba y se les solicitó que calificaran en una escala del 1 al 6 su grado de acuerdo con el ítem correspondiente al factor que había sido asignado. El análisis de validez de contenido se realizó utilizando la prueba Chi cuadrado y también el método de Aiken (1996). Todos los ítems obtuvieron coeficientes superiores a los señalados al nivel de 0.01 . Al finalizar esta etapa se procedió a construir la versión final de la prueba, no se eliminó ningún ítem. La versión final del instrumento quedó conformada por 56 ítems.

\section{Cuestionario sobre las fuentes de presión laboral}

FICHA TÉCNICA:

Nombre:

Autor

Año de publicación

Administración:

Duración

Aplicación

Significación

Factores que mide

Número de ítems

Normas

Validez y confiabilidad
Inventario de presiones a las que se enfrenta el docente

: Manuel Fernández Arata

: 2001

: Individual y grupal

15 a 20 minutos.

Docentes

: Identificar las fuentes de presión para toda persona en un momento dado y la forma como los individuos perciben esas fuentes potenciales de presión

: I. Presiones sociales derivadas de la labor educativa en el aula.

II. Presiones derivadas de la vida cotidiana en el aula.

III. Presiones originadas por la organización administrativa.

IV. Flexibilidad con respecto a la tarea docente.

: 84

Percentilares

: La confiabilidad obtenida a través del Alfa de Cronbach fue de 0.96 (factor I), 0.94 (factor II), 0.91 (factor III) y 0.81 (factor IV).

Se obtuvo la validez de constructo $(0.94)$ y de contenido $(0.89$ a 1.00$)$. 


\section{TÉCNICAS DE RECOLECCIÓN DE DATOS}

Para aplicar los instrumentos se solicitó a los decanos de las distintas facultades de las universidades seleccionadas la autorización respectiva. Posteriormente, se solicitó una entrevista con los respectivos decanos o sus representantes para agilizar la autorización. En la totalidad de casos esta fue otorgada rápidamente, mostrándose buena disposición hacia el proyecto.

La recolección de datos se realizó mediante la aplicación directa e individual de los instrumentos a los docentes de las diferentes facultades de las universidades seleccionadas. Los obstáculos que se presentaron para la aplicación de los instrumentos fueron múltiples: la ubicación de los docentes según facultad, la aceptación para responder a los instrumentos y la devolución de las respuestas. Asimismo, la paralización de actividades por más de cuatro meses en algunas universidades.

El periodo de aplicación comprendió un lapso de 16 meses, entre julio del 2003 y octubre del 2004.

\section{REsultados}

En primer lugar, se realizó el análisis psicométrico de los instrumentos de recolección de datos y, posteriormente, el análisis de las variables estudiadas.

La confiabilidad de la Escala de Satisfacción Docente fue estimada mediante la técnica de la consistencia interna, utilizando el coeficiente Alfa de Cronbach. La validez de constructo se estimó mediante el análisis factorial confirmatorio.

Los valores del coeficiente Alfa de Cronbach del factor intrínseco ascendió a 0.82 , el factor extrínseco a 0.82 y los coeficientes de los diferentes subfactores fluctuaron entre 0.72 y 0.88 . El análisis de la confiabilidad generalizado asciende a 0.93. Estos resultados permiten afirmar que los factores, subfactores e ítems que miden la satisfacción laboral permiten obtener puntajes confiables.

El estudio de la validez de constructo de la Escala de Satisfacción Laboral fue realizado a través del Análisis Factorial Confirmatorio, aplicándose el programa Amos 5.0 (Arbuckle, 2000, 2004). Los resultados alcanzados en el modelo teórico propuesto de 11 subfactores, de acuerdo con las recomendaciones de Byrne (2001), se contrastó con dos modelos alternativos: el modelo saturado (todas las variables están correlacionadas entre sí) y el modelo independiente (donde las variables son totalmente independientes entre sí). Los resultados del análisis de la Escala de Satisfacción permiten concluir que esta presenta validez de constructo.

En relación con el inventario de presiones a las que se enfrenta el docente fue necesario evaluar la confiabilidad, en razón de las posibles variaciones al aplicarse un instrumento en diferentes muestras (Muñiz, 1994, 
1996). El análisis de ítems permitió que los índices de discriminación de los ítems fluctuaran entre 0.41 y 0.74 superiores al valor considerado como mínimo aceptable (0.20); el índice de discriminación total obtenido asciende a 0.94. Los valores del coeficiente Alfa de Cronbach del factor Presiones sociales derivadas de la labor educativa en el aula ascendió a 0.96 , en el factor Presiones derivadas de la vida cotidiana en el aula ascendió a 0.94, en el factor Presiones originadas por la organización administrativa ascendió a 0.91 y el factor Flexibilidad con respecto a la tarea docente correspondió a 0.81 . Estos resultados permiten afirmar que los factores e ítems que miden las fuentes de presión laboral posibilitan obtener puntajes confiables.

El estudio de la validez de constructo de la Escala de Presión Laboral fue realizado, igualmente, mediante el Análisis Factorial Confirmatorio, aplicándose el programa Amos 5.0 (Arbuckle, 2000, 2004). Los resultados alcanzados en el modelo teórico propuesto de un factor, de acuerdo con las recomendaciones de Byrne (2001), se contrastó con dos modelos alternativos: el modelo saturado (todas las variables están correlacionadas entre sí) y el modelo independiente (las variables son totalmente independientes entre sí). Los resultados permiten concluir que la Escala de Presión Laboral presenta validez de constructo.
Para realizar el análisis estadístico se inició el análisis de asunción de normalidad mediante la prueba estadística de normalidad. Para tal fin, el análisis de la bondad de ajuste a la curva normal se realizó mediante el test de Kolmogorov-Smirnov, cuyos resultados, indicaron que los factores intrínseco y extrínseco de la Escala de Satisfacción Docente y los cuatro factores del inventario de presiones a las que se enfrenta el docente presentan significación estadística, por lo que se puede concluir que las distribuciones de las variables analizadas se aproximan a la curva normal. Estos resultados permitieron la utilización de estadísticas de tipo paramétrico en análisis de los datos.

\section{ANÁLISIS DE LAS VARIABLES ESTUDIADAS \\ Relación entre la Satisfacción Laboral y las Fuentes de Presión Laboral}

El resultado del análisis de correlación permite observar (tabla 4) que los factores intrínseco y extrínseco y el puntaje total de la Escala de la Satisfacción Laboral muestran correlación negativa, al nivel de significación de 0.001 , con los factores Presiones sociales derivadas de la labor educativa en el aula, Presiones derivadas de la vida cotidiana en el aula, Presiones originadas por la organización administrativa, Flexibilidad con respecto a la tarea docente y puntaje total del Inventario de la Presión Laboral. 
Tabla 4

Matriz de correlaciones entres satisfacción laboral y presión laboral

\begin{tabular}{llllll}
\hline \multicolumn{1}{c}{ Satisfacción laboral } & \multicolumn{5}{l}{ Presión laboral } \\
\hline & Factor 1 & Factor 2 & Factor 3 & Factor 4 & Total \\
\hline Intrínseco & $-0.31^{* * *}$ & $-0.30^{* * *}$ & $-0.34^{* * *}$ & $-0.16^{* * *}$ & $-0.31^{* * *}$ \\
Extrínseco & $-0.28^{* * *}$ & $-0.22^{* * *}$ & $-0.34^{* * *}$ & $-0.11^{*}$ & $-0.26^{* * *}$ \\
Total & $-0.31^{* * *}$ & $-0.28^{* * *}$ & $-0.35^{* * *}$ & $-0.14^{* *}$ & $-0.30^{* * *}$ \\
\hline
\end{tabular}

${ }^{*} p<.05^{* *} p<.011^{* * *} p<.001 \quad n=506$

Estos resultados permiten afirmar que las Fuentes de Presión Laboral y la Satisfacción Laboral se relacionan de manera inversa en los docentes universitarios de Lima metropolitana.

Relaciones entre los subfactores de la Escala de Satisfacción Docente y las Fuentes de Presión Laboral
La tabla 5 nos permite observar que todos los subfactores de la Escala de Satisfacción Laboral correlacionan negativamente con los cuatro factores del inventario de las Fuentes de Presión Laboral. Es decir, se establece una relación inversa entre los subfactores de la Escala de Satisfacción Laboral y los factores del inventario de presiones a las que se enfrenta el docente.

Tabla 5

Matriz de correlaciones entre los subfactores de las Escalas de Satisfacción Laboral y las Fuentes de Presión Laboral

\begin{tabular}{|c|c|c|c|c|c|}
\hline & \multicolumn{5}{|c|}{ Presión laboral } \\
\hline & Factor 1 & Factor 2 & Factor 3 & Factor 4 & Total \\
\hline Independencia & $-0.15^{\star *}$ & $-0.19^{* * *}$ & $-0.09^{*}$ & -0.07 & $-0.14^{\star *}$ \\
\hline Variedad & $-0.11^{*}$ & $-0.15^{\star *}$ & $-0.15^{\star *}$ & -0.03 & $-0.12^{* *}$ \\
\hline Libertad de cátedra & $-0.26^{\star * *}$ & $-0.37^{* * *}$ & $-0.23^{* * *}$ & $-0.20^{* *}$ & $-0.30^{* * *}$ \\
\hline Línea de carrera & $-0.32^{* * *}$ & $-0.25^{\star * *}$ & $-0.36^{* * *}$ & $-0.18^{* * *}$ & $-0.31^{* \star *}$ \\
\hline Reconocimiento y distinciones & $-0.31^{\star \star *}$ & $-0.23^{* * *}$ & $-0.39^{* * *}$ & $-0.14^{\star *}$ & $-0.30^{* *}$ \\
\hline Compensación económica & $-0.21^{* * *}$ & $-0.12^{* *}$ & $-0.33^{* * *}$ & $-0.11^{*}$ & $-0.21^{* * *}$ \\
\hline Condiciones de trabajo & $-0.26^{* * *}$ & $-0.22^{\star * *}$ & $-0.25^{\star * *}$ & $-0.09^{*}$ & $-0.23^{* * *}$ \\
\hline Seguridad en el puesto & $-0.12^{* \star}$ & $-0.19^{\star * *}$ & $-0.16^{* * *}$ & $-0.12^{* \star}$ & $-0.17^{\star \star *}$ \\
\hline Estatus & $-0.12^{* *}$ & $-0.14^{\star *}$ & $-0.10^{*}$ & -0.01 & $-0.10^{*}$ \\
\hline Calidad de relaciones interpersonales & $-0.22^{* * *}$ & $-0.15^{\star *}$ & $-0.24^{\star * *}$ & -0.01 & $-0.17^{* * *}$ \\
\hline Capacitación y perfeccionamiento & $-0.27^{* * *}$ & $-0.16^{\star * *}$ & $-0.34^{* * *}$ & $-0.12^{\star *}$ & $-0.25^{\star * *}$ \\
\hline
\end{tabular}


Asimismo, se estudió la relación entre los conjuntos de variables que pueden asumirse como variables independientes (predictoras) y su influencia en las variables dependientes (criterio) mediante la correlación canónica.

El resultado del análisis de correlación canónica, presentado en la tabla 6 , permite observar la presencia de dos variables canónicas que presentan correlaciones estadísticas significativas. Pero al analizar la varianza explicada y la redundancia de ambas variables, es factible indicar que, tanto para el caso del grupo 1 de variables (Presión Laboral) como para el grupo 2 de variables (Satisfacción) los valores más ele- vados corresponden a la primera variable canónica, motivo por el cual -en términos conceptuales- es más conveniente analizarla con mayor profundidad.

La primera variable canónica alcanza un valor de correlación canónica (entre las variables de los grupos 1 y 2) que puede ser clasificada como alta, pues asciende a 0.70 . También se aprecia que las variables del grupo 1 correlacionan de forma similar con la primera variable canónica, pues todas son negativas y mayores de 0.60 . De otro lado, las variables del grupo 2 correlacionan de forma positiva y mayor de 0.90 con la primera variable canónica.

Tabla 6

Análisis de correlación canónica entre los grupos de variables estudiadas de la Presión Laboral y Satisfacción Laboral

\begin{tabular}{|c|c|c|c|c|}
\hline \multirow[b]{2}{*}{ Variable } & \multicolumn{2}{|c|}{ Primera variable canónica } & \multicolumn{2}{|c|}{ Segunda variable canónica } \\
\hline & Correlación & $\begin{array}{c}\text { Coeficiente } \\
\text { canónico }\end{array}$ & Correlación & $\begin{array}{c}\text { Coeficiente } \\
\text { canónico }\end{array}$ \\
\hline Grupo 1 de variables & -0.81 & -0.15 & -0.35 & 0.04 \\
\hline Presión Laboral 1 & -0.71 & -0.06 & -0.71 & -1.32 \\
\hline Presión Laboral 2 & -0.95 & -1.11 & -0.12 & 1.17 \\
\hline Presión Laboral 3 & -0.64 & 0.32 & -0.55 & -0.32 \\
\hline \multicolumn{5}{|l|}{ Presión Laboral 4} \\
\hline Varianza explicada & \multicolumn{2}{|c|}{0.65} & \multicolumn{2}{|c|}{0.23} \\
\hline Redundancia & \multicolumn{2}{|c|}{0.09} & \multicolumn{2}{|c|}{0.01} \\
\hline Grupo 2 de variables & 0.93 & 0.59 & 0.38 & 2.57 \\
\hline $\begin{array}{l}\text { Satisfacción Intrínseca } \\
\text { Satisfacción Extrínseca }\end{array}$ & 0.97 & 1.05 & -0.23 & -2.55 \\
\hline Varianza explicada & \multicolumn{2}{|c|}{0.90} & \multicolumn{2}{|c|}{0.10} \\
\hline Redundancia & \multicolumn{2}{|c|}{0.12} & \multicolumn{2}{|c|}{0.01} \\
\hline Correlación Canónica & \multicolumn{2}{|c|}{0.70} & \multicolumn{2}{|c|}{0.22} \\
\hline Chi-Cuadrado & \multicolumn{2}{|c|}{$100.66^{* * *}$} & \multicolumn{2}{|c|}{$26.71^{* * *}$} \\
\hline
\end{tabular}

${ }^{*} p<.05$ ** $p<.01$ *** $p<.001 \quad n=506$ 
Satisfacción Laboral y Fuentes de Presión Laboral según tipo de gestión

En la tabla 7 apreciamos que en la Escala de Satisfacción Laboral los docentes de las universidades privadas obtienen mayores puntajes promedios en el factor extrínseco, intrínseco y la escala total. Al realizar las comparaciones entre ambos grupos se aprecia que existen diferencias estadísticamente significativas.
La tabla 8 muestra que en la Escala de Fuentes de Presión Laboral los docentes de las universidades estatales obtienen mayores promedios en los cuatro factores. Al realizar las comparaciones entre ambos grupos se aprecia que existen diferencias estadísticamente significativas en estos, excepto en el factor presiones sociales derivadas de la labor educativa en el aula.

Tabla 7

Comparación de la Satisfacción Laboral según tipo de gestión

\begin{tabular}{lccccc}
\hline & \multicolumn{2}{c}{$\begin{array}{c}\text { Universidad estatal } \\
n=212\end{array}$} & \multicolumn{2}{c}{ Universidad privada } & $\mathrm{Z}$ \\
& $\mathrm{M}$ & $\mathrm{D}=294$ & $\mathrm{M}$ & $\mathrm{D} . \mathrm{E}$ & \\
\hline Intrínseca & 4.03 & 0.58 & 4.48 & 0.64 & $-8.09^{* * *}$ \\
Extrínseca & 3.62 & 0.40 & 4.23 & 0.63 & $-12.36^{* * *}$ \\
Total & 3.80 & 0.44 & 4.34 & 0.62 & $-10.88^{* * *}$ \\
\hline
\end{tabular}

${ }^{*} p<.05$

Tabla 8

Prueba $Z$ de la diferencia de medias de la comparación de la Fuentes de Presión Laboral según tipo de gestión

\begin{tabular}{|c|c|c|c|c|c|}
\hline \multirow[t]{2}{*}{ Presión laboral } & \multicolumn{2}{|c|}{$\begin{array}{l}\text { Universidad estatal } \\
\qquad n=212\end{array}$} & \multicolumn{2}{|c|}{$\begin{array}{l}\text { Universidad privada } \\
\qquad \mathrm{n}=294\end{array}$} & \multirow[t]{2}{*}{ Z } \\
\hline & $\mathrm{M}$ & D.E & $\mathrm{M}$ & D.E. & \\
\hline $\begin{array}{l}\text { I. Derivadas de la labor } \\
\text { educativa en el aula }\end{array}$ & 3.65 & 0.91 & 3.25 & 1.22 & 1.62 \\
\hline $\begin{array}{l}\text { II. Derivadas de la vida } \\
\text { cotidiana en el aula }\end{array}$ & 3.42 & 1.20 & 3.19 & 0.90 & $5.66^{\star \star \star}$ \\
\hline $\begin{array}{l}\text { III. Organización } \\
\text { administrativa }\end{array}$ & 3.29 & 1.04 & 3.11 & 0.95 & $2.07^{*}$ \\
\hline $\begin{array}{l}\text { IV. Flexibilidad respecto } \\
\text { a la tarea docente }\end{array}$ & 3.29 & 0.91 & 2.81 & 1.03 & $5.39^{* * *}$ \\
\hline Total & 3.41 & 0.89 & 3.09 & 0.90 & $4.02^{\star \star \star}$ \\
\hline
\end{tabular}


Subfactores de la Satisfacción Laboral y tipo de gestión

La tabla 9 nos permite apreciar que en los 11 subfactores, los docentes de las universidades privadas obtienen puntajes totales promedios mayores que los docentes de universidades estatales. Al realizar las comparaciones respectivas se observa que existen diferencias significativas en diez de los once subfactores, notándose que solo en el subfactor Seguridad en el puesto no muestra diferencias.

\section{Influencia de los factores de las}

Fuentes de Presión Laboral sobre los factores de la Satisfacción Laboral

Para el contraste se aplicó el análisis multivariado del Modelo de Ecuaciones Estructurales, en su modalidad de análisis de senderos (Path análisis) que se realizó sobre la base de la aplicación del programa AMOS 5.0 (Arbuckle, 2000, 2004), tomándose en cuenta las recomendaciones de Byrne (2001), quien propone la necesidad de evaluar el modelo teórico propuesto y dos modelos alternativos diferenciados que sirvan de criterio de contraste.

En primer lugar, las Fuentes de Presión Laboral fueron consideradas como variables independientes y los factores de la Satisfacción Laboral como variables dependientes, estableciéndose las relaciones entre todas las variables independientes con todas las variables dependientes, los resultados indicaron que el modelo no fue adecuado $\left(X^{2}{ }_{(6)}=1439.22 \mathrm{p}<.0001\right)$, por ello se procedió a eliminar las relaciones

Tabla 9

Prueba $Z$ de diferencia de medias de la comparación de la Satisfacción Laboral según tipo de gestión

\begin{tabular}{|c|c|c|c|c|c|}
\hline \multirow[t]{2}{*}{ Satisfacción laboral } & \multicolumn{2}{|c|}{$\begin{array}{l}\text { Universidad estatal } \\
\qquad n=212\end{array}$} & \multicolumn{2}{|c|}{$\begin{array}{l}\text { Universidad privada } \\
\qquad \mathrm{n}=294\end{array}$} & \multirow[t]{2}{*}{ Z } \\
\hline & $\mathrm{M}$ & D.E & $\mathrm{M}$ & D.E. & \\
\hline Independencia & 4.7107 & .73156 & 4.9785 & .74504 & $-4.02^{* * *}$ \\
\hline Variedad & 4.0453 & .75226 & 4.4395 & .73457 & $-5.90^{* * *}$ \\
\hline Libertad de cátedra & 4.3500 & .73191 & 4.5803 & .73509 & $-3.48^{\star *}$ \\
\hline Línea de carrera & 3.6934 & .92329 & 4.2327 & .90158 & $-6.57^{\star * *}$ \\
\hline Reconocimiento y distinciones & 3.3358 & .93952 & 4.1558 & .95757 & $-9.58^{* * *}$ \\
\hline Compensación económica & 2.7698 & .71929 & 3.8694 & .88988 & $-14.33^{\star * *}$ \\
\hline Condiciones de trabajo & 3.7613 & .55367 & 4.2993 & .72040 & $-9.11^{* * *}$ \\
\hline Seguridad en el puesto & 3.8137 & .69618 & 3.9328 & .72935 & -1.85 \\
\hline Estatus & 4.5642 & .77020 & 4.7857 & .78723 & $-3.15^{\star *}$ \\
\hline Calidad de relaciones interpersonales & 3.6198 & .79321 & 4.2531 & .87882 & $-8.33^{\star * *}$ \\
\hline Capacitación y perfeccionamiento & 3.1623 & .83643 & 4.2211 & .89624 & $-13.48^{* * *}$ \\
\hline
\end{tabular}

** $p<.01^{* * *} p<.001$ 
entre las variables independientes y dependientes que no eran significativas, hasta llegar al modelo final.

En los resultados observados en la tabla 10, donde se incluye el modelo teórico propuesto que indica la existencia de relaciones implicativas entre las variables independientes y las variables dependientes, se puede observar que el modelo propuesto alcanza en el test de Bondad de Ajuste ChiCuadrado Mínimo $\left(X^{2}\right)$ un valor de 0.524 , que con ocho grados de libertad alcanza una probabilidad de 0.770 , lo que nos indica que el modelo es adecuado y obtiene resultados superiores a los modelos Saturado e Independiente.

La revisión de los hallazgos de los análisis complementarios de la Bondad de Ajuste a través del Índice de Ajuste $(\mathrm{GFI}=1.000)$ y el Índice de Ajuste Ponderado (AGFI $=0.996)$ alcanzaron valores óptimos. Además, el análisis del índice residual de la raíz cuadrada media del error de aproximación, que evalúa la aproximación de la matriz de covarianza teórica con la matriz observada, alcanzó un valor pequeño $($ RMSEA $=0.001)$.

Estos hallazgos permitieron corroborar lo pertinente de aceptar el modelo propuesto y, por lo tanto, los Factores de las Fuentes de Presión Laboral influyen sobre los Factores de la Satisfacción Laboral.

El análisis estadístico de las influencias específicas de las variables independientes sobre las variables dependientes, planteadas en el modelo y que son analizadas a través de la Razón Crítica (RC), presentadas en las tabla 11 y la figura 1 , permiten apreciar que las relaciones propuestas son estadísticamente significativas y por lo tanto son relevantes para conformar el modelo. Dichas relaciones son:

Tabla 10

Análisis del modelo de ecuaciones estructurales de la influencia de la Presión Laboral sobre la Satisfacción Laboral

\begin{tabular}{lccc}
\hline Datos & Modelo evaluado & Modelo saturado & Modelo independiente \\
\hline Parámetros & 19 & 21 & 6 \\
Chi-cuadrado mínimo & 0.524 & 0.000 & 2091.286 \\
G. L. & 8 & 0 & 15 \\
P & .770 & & .0001 \\
Chi-cuadrado mínimo/G.L. & 0.066 & 1.000 & 139.419 \\
GFI & 1.000 & & 0.414 \\
AGFI & 0.996 & & 0.179 \\
RMSEA & 0.001 & & 0.524
\end{tabular}

$n=506$ 
a) La Presión Laboral 1, derivada de la labor educativa en el aula, influye negativamente sobre la Satisfacción Extrínseca $(\beta=-0.15)$.

b) La Presión Laboral 2, derivada de la vida cotidiana en el aula, influye negativamente sobre la Satisfacción Intrínseca $(\beta=-0.18)$.

c) La Presión Laboral 3, originada por la organización administrativa, influye negativamente sobre la Satisfacción Extrínseca $(\beta=-0.42)$. d) La Presión Laboral 3, originada por la organización administrativa, influye negativamente sobre la Satisfacción Intrínseca $(\beta=-0.27)$.

e) La Presión Laboral 4, flexibilidad con respecto a la tarea docente, influye positivamente sobre la Satisfacción Extrínseca $(\beta=0.16)$.

f) La Presión Laboral 4, flexibilidad con respecto a la tarea docente, influye positivamente sobre la Satisfacción Intrínseca $(\beta=0.12)$.

Figura 1

Modelo de ecuaciones estructurales de la influencia de la Presión Laboral sobre los Factores de la Satisfacción Laboral

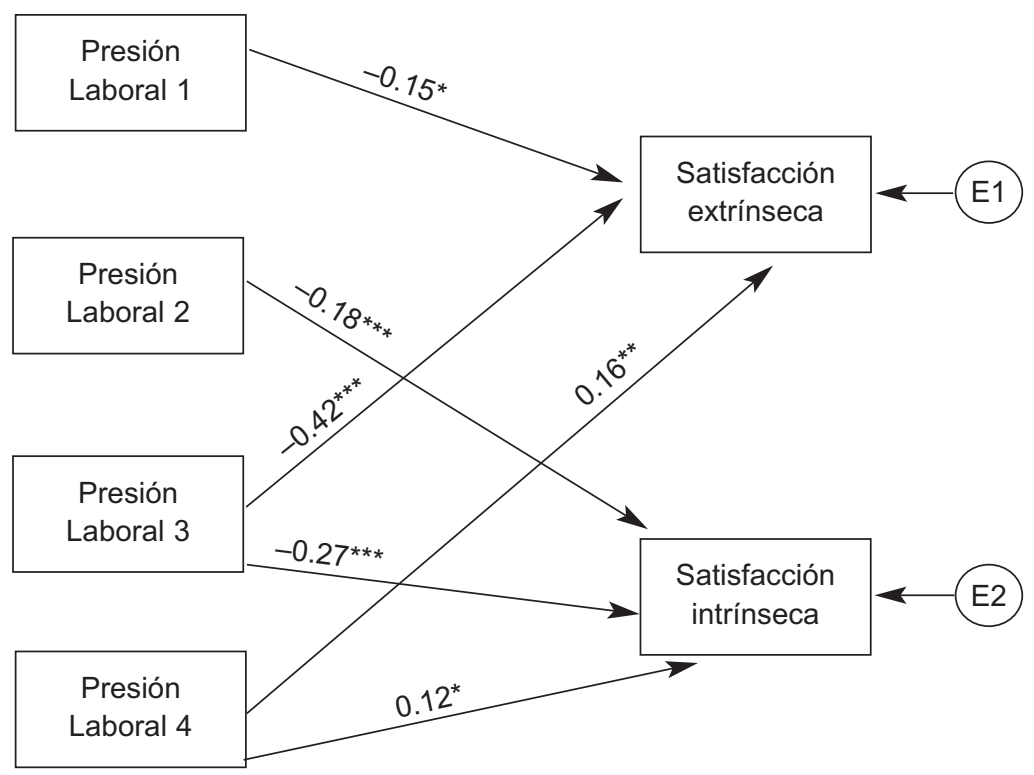

Modelo de ecuaciones estructurales

$\begin{array}{lll}\text { Chi cuadrado } & = & 0.524 \\ \text { G.L. } & = & 8 \\ \mathrm{p} & = & .770\end{array}$

${ }^{*}<.05 \quad{ }^{* *} p<.01 \quad * * * p<.001$ 
Tabla 11

Análisis de la influencia de la Presión Laboral sobre la Satisfacción Laboral en el Modelo de Ecuaciones Estructurales

\begin{tabular}{|c|c|c|c|c|c|}
\hline & V.I. & V.D: & $\begin{array}{c}\beta \\
\text { (Beta estandarizado) }\end{array}$ & Razón crítica & $\mathrm{p}$ \\
\hline 1 & Presión Laboral 1 & Satisfacción Extrínseca & -0.15 & -2.277 & .0410 \\
\hline 2 & Presión Laboral 2 & Satisfacción Intrínseca & -0.18 & -4.158 & .0001 \\
\hline 3 & Presión Laboral 3 & Satisfacción Extrínseca & -0.42 & -6.640 & .0001 \\
\hline 4 & Presión Laboral 3 & Satisfacción Intrínseca & -0.27 & -4.556 & .0001 \\
\hline 5 & Presión Laboral 4 & Satisfacción Extrínseca & 0.16 & 3.035 & .0020 \\
\hline 6 & Presión Laboral 4 & Satisfacción Intrínseca & 0.12 & 2.160 & .0310 \\
\hline
\end{tabular}

$N=506$

Satisfacción Laboral y Fuentes de Presión Laboral según el ejercicio de la docencia en pregrado y posgrado

La tabla 12 muestra que en la variable Ejercicio de la Docencia en Pregrado o Posgrado, los docentes que trabajan en pregrado obtienen mayores puntajes promedios en los factores Intrínseco,
Extrínseco y Total que aquellos que laboran en posgrado.

Igualmente, en todos los subfactores de la escala los docentes que laboran en pregrado obtienen mayor puntaje promedio que los que trabajan en posgrado, excepto en el subfactor Línea de Carrera. Al realizar las res-

Tabla 12

Prueba $Z$ de la diferencia de medias comparación de la Satisfacción Laboral según ejercicio de la docencia

\begin{tabular}{lrrrrc}
\hline \multirow{2}{*}{ Satisfacción laboral } & \multicolumn{2}{c}{$\begin{array}{c}\text { Pregrado } \\
\mathrm{n}=480\end{array}$} & \multicolumn{2}{c}{ Postgrado } & $\mathrm{Z}=26$ \\
& Media & D.E. & Media & D.E. \\
\hline Intrínseca & 4.30 & 0.66 & 4.07 & 0.57 & 1.76 \\
Extrínseca & 3.98 & 0.63 & 3.72 & 0.41 & $2.10^{*}$ \\
Total & 4.13 & 0.62 & 3.88 & 0.42 & $2.04^{*}$ \\
Independencia & 4.88 & 0.74 & 4.55 & 0.94 & $2.21^{*}$ \\
Variedad & 4.29 & 0.76 & 3.92 & 0.82 & $2.41^{*}$ \\
Libertad de cátedra & 4.50 & 0.73 & 4.11 & 0.87 & $2.62^{* *}$ \\
Línea de carrera & 4.01 & 0.96 & 4.03 & 0.80 & -0.13 \\
Reconocimiento y distinciones & 3.82 & 1.04 & 3.72 & 0.79 & 0.45 \\
Compensación económica & 3.42 & 0.99 & 3.15 & 0.84 & 1.40 \\
Condiciones de trabajo & 4.08 & 0.71 & 3.88 & 0.55 & 1.40 \\
Seguridad en puesto & 3.89 & 0.71 & 3.77 & 0.77 & 0.83 \\
Estatus & 4.71 & 0.79 & 4.70 & 0.79 & 1.85 \\
Calidad de relaciones interpersonales & 4.01 & 0.90 & 3.62 & 0.79 & $2.18^{*}$ \\
Capacitación y perfeccionamiento & 3.79 & 1.03 & 3.49 & 0.70 & 1.47 \\
\hline
\end{tabular}

${ }^{*} p<.05{ }^{* *} p<.01$ 
pectivas comparaciones, se aprecia que se presentan diferencias estadísticamente significativas en los factores extrínsecos $(Z=2.10 \mathrm{p}<0.05)$, Total $(Z=2.04 \mathrm{p}<0.05)$, Independencia $(\mathrm{Z}=2.21 \mathrm{p}<0.05)$, Variedad $(\mathrm{Z}=2.41$ $\mathrm{p}<0.05)$, Libertad de cátedra $(Z=2.62$ $\mathrm{p}<0.01)$ y Calidad de relaciones interpersonales $(Z=2.18 \mathrm{p}<0.05)$.
La tabla 13 muestra que no se encuentran diferencias estadísticamente significativas en las Fuentes de Presión Laboral entre los docentes que laboran en pregrado y posgrado.

La tabla 14 muestra que los docentes que laboran en la condición de contratados obtienen mayores promedios estadísticamente significativos en los

Tabla 13

Comparación de la Presión laboral según el ejercicio de la docencia

\begin{tabular}{lrrrrr}
\hline & \multicolumn{2}{c}{$\begin{array}{c}\text { Pregrado } \\
\mathrm{n}=480\end{array}$} & \multicolumn{2}{c}{$\begin{array}{c}\text { Postgrado } \\
\mathrm{n}=269\end{array}$} & Z \\
& Media & D.E. & Media & D.E. & \\
\hline I. Derivadas de la labor educativa en el aula laboral & 3.01 & 1.01 & 3.08 & 1.05 & -0.35 \\
$\begin{array}{l}\text { II. Derivadas de la vida educativa cotidiana } \\
\quad \text { en el aula }\end{array}$ & 3.18 & 0.99 & 3.28 & 1.02 & -0.51 \\
III. Organización administrativa & 3.38 & 0.93 & 3.39 & 0.97 & -0.03 \\
IV. Flexibilidad respecto a la tarea docente & 3.33 & 1.21 & 3.14 & 1.28 & 0.76 \\
Total & 3.22 & 0.90 & 3.22 & 1.01 & 0.01 \\
\hline
\end{tabular}

Tabla 14

Prueba Z de la diferencia de medias de la comparación de la Satisfacción Laboral según condición

\begin{tabular}{lccccc}
\hline \multirow{2}{*}{ Satisfacción laboral } & \multicolumn{2}{c}{$\begin{array}{c}\text { Nombrado } \\
\mathrm{n}=312\end{array}$} & \multicolumn{2}{c}{ Contratado } \\
$\mathrm{n}=193$ & $\mathrm{Z}$ \\
& Media & D.E. & Media & D.E. & \\
\hline Intrínseca & 4.26 & 0.66 & 4.34 & 0.66 & -1.45 \\
Extrínseca & 3.94 & 0.61 & 4.02 & 0.65 & -1.25 \\
Total & 4.09 & 0.60 & 4.16 & 0.63 & -1.41 \\
Independencia & 4.82 & 0.75 & 4.93 & 0.75 & -1.52 \\
Variedad & 4.25 & 0.77 & 4.31 & 0.76 & 0.88 \\
Libertad de cátedra & 4.46 & 0.76 & 4.53 & 0.71 & -0.94 \\
Línea de carrera & 4.01 & 0.98 & 4.01 & 0.90 & -0.07 \\
Reconocimiento y distinciones & 3.74 & 1.02 & 3.93 & 1.05 & $-2.10^{*}$ \\
Compensación económica & 3.35 & 1.00 & 3.51 & 0.95 & -1.79 \\
Condiciones de trabajo & 4.03 & 0.68 & 4.14 & 0.75 & -1.58 \\
Seguridad en puesto & 3.97 & 0.69 & 3.74 & 0.73 & $3.44^{* *}$ \\
Estatus & 4.71 & 0.76 & 4.67 & 0.84 & 0.45 \\
Calidad de relaciones interpersonales & 3.91 & 0.91 & 4.11 & 0.87 & -2.50 * \\
Capacitación y perfeccionamiento & 3.70 & 1.07 & 3.91 & 0.91 & -2.34 * \\
\hline
\end{tabular}

${ }^{*} p<.05{ }^{* *} p<.01$ 
subfactores Reconocimiento y Distinciones $(Z=-2.10 p<0.05)$, Calidad de relaciones interpersonales $(Z=$ $-2.50 \mathrm{p}<0.05)$ y Capacitación y perfeccionamiento docente $(\mathrm{Z}=-2.34 \mathrm{p}<$ 0.05 ); en tanto que los docentes nombrados obtienen mayores puntajes estadísticamente significativos en el subfactor Seguridad en el puesto $(\mathrm{Z}=$ $3.44 \mathrm{p}<0.01)$.

La tabla 15 muestra que no se encuentran diferencias estadísticamente significativas en los diferentes factores del inventario de las Fuentes de Presión Laboral entre los docentes que laboran en la condición de nombrados y contratados.

\section{Satisfacción Laboral y Fuentes de Presión Laboral según la dedicación}

La tabla 16 muestra que se encuentran diferencias significativas entre los docentes que laboran a tiempo completo y los docentes que lo hacen a dedicación exclusiva, y entre los docentes a tiempo parcial y los que están a dedicación exclusiva en los factores intrínseco, extrínseco y escala total.

Asimismo, se encuentran diferencias significativas entre los docentes a tiempo completo y a dedicación exclusiva, y los docentes que prestan servicios a tiempo parcial y los que laboran a dedicación exclusiva, en los subfactores Compensación económica, Condiciones de trabajo, Seguridad en el puesto, Calidad de relaciones interpersonales y Capacitación y perfeccionamiento docente.

La tabla 17 muestra que los docentes que laboran a dedicación exclusiva obtienen mayores promedios estadísticamente significativos en los factores derivados de la labor educativa en el aula y de la organización administrativa.

Tabla 15

Comparación de la Presión laboral según condición

\begin{tabular}{lrrrrc}
\hline & \multicolumn{2}{c}{$\begin{array}{c}\text { Nombrado } \\
n=312\end{array}$} & \multicolumn{2}{c}{$\begin{array}{c}\text { Contratado } \\
n=193\end{array}$} & Z \\
Presión laboral & Media & D.E. & Media & D.E. & \\
\hline I. Derivadas de la labor educativa en el aula & 3.02 & 1.03 & 2.99 & 0.98 & 0.40 \\
$\begin{array}{l}\text { II. Derivadas de la vida educativa cotidiana } \\
\quad \text { en el aula }\end{array}$ & 3.19 & 1.05 & 3.17 & 0.89 & 0.29 \\
III. Organización administrativa & 3.43 & 0.95 & 3.31 & 0.89 & 1.38 \\
IV. Flexibilidad respecto a la tarea docente & 3.28 & 1.28 & 3.38 & 1.09 & 0.94 \\
Total & 3.23 & 0.96 & 3.21 & 0.83 & 0.23 \\
\hline
\end{tabular}


Tabla 16

Comparación de la Satisfacción Laboral según dedicación

\begin{tabular}{lcrcc}
\hline Satisfacción laboral & $\begin{array}{c}\text { Tiempo } \\
\text { completo } \\
\mathrm{N}=189 \\
\text { Media }\end{array}$ & $\begin{array}{c}\text { Tiempo } \\
\text { parcial } \\
\mathrm{N}=240 \\
\text { Media. }\end{array}$ & $\begin{array}{c}\text { Dedicación } \\
\text { exclusiva } \\
\mathrm{N}=73 \\
\text { Media }\end{array}$ & $\mathrm{F}$ \\
\hline Intrínseca & $4.34 \mathrm{a}$ & 4.30 & $4.12 \mathrm{a}$ & $3.32^{*}$ \\
Extrínseca & $4.00 \mathrm{a}$ & $4.02 \mathrm{~b}$ & $3.75 \mathrm{ab}$ & $5.45^{* *}$ \\
Total & $4.16 \mathrm{a}$ & $4.15 \mathrm{~b}$ & $3.92 \mathrm{ab}$ & $4.72^{*}$ \\
Independencia & 4.86 & 4.92 & 4.70 & 2.54 \\
Variedad & 4.31 & 4.28 & 4.18 & 0.75 \\
Libertad de cátedra & 4.54 & 4.47 & 4.42 & 0.87 \\
Línea de carrera & $4.16 \mathrm{a}$ & 3.97 & $3.77 \mathrm{a}$ & 5.04 \\
Reconocimiento y distinciones & 3.85 & $3.88 \mathrm{~b}$ & $3.51 \mathrm{~b}$ & 3.74 \\
Compensación económica & $3.49 \mathrm{a}$ & $3.49 \mathrm{~b}$ & $2.94 \mathrm{ab}$ & $10.32^{* * *}$ \\
Condiciones de trabajo & 4.11 & $4.11 \mathrm{~b}$ & $3.87 \mathrm{~b}$ & $3.59^{*}$ \\
Seguridad en el puesto & 3.95 & $3.78 \mathrm{~b}$ & $4.08 \mathrm{~b}$ & $6.01^{* *}$ \\
Estatus & 4.71 & 4.69 & 4.68 & 0.04 \\
Calidad de las relaciones interpersonales & 3.99 & $4.07 \mathrm{~b}$ & $3.73 \mathrm{~b}$ & $3.94^{*}$ \\
Capacitación y perfeccionamiento & $3.78 \mathrm{a}$ & $3.96 \mathrm{~b}$ & $3.22 \mathrm{ab}$ & $15.69^{* * *}$ \\
\hline
\end{tabular}

${ }^{*} p<.05{ }^{* *} p<.01{ }^{* * *} p<.001$

Las diferencias por pares de grupos se realizaron a través del test de Scheffé: a, b, c.

Tabla 17

Tabla de la varianza de la comparación de la Presión Laboral según dedicación

\begin{tabular}{lrrrr}
\hline Presión laboral & $\begin{array}{c}\text { Tiempo } \\
\text { completo } \\
\mathrm{N}=189 \\
\text { Media }\end{array}$ & $\begin{array}{c}\text { Tiempo } \\
\text { parcial } \\
\mathrm{N}=240 \\
\text { Media }\end{array}$ & $\begin{array}{c}\text { Dedicación } \\
\text { exclusiva } \\
\mathrm{N}=74 \\
\text { Media }\end{array}$ & $\mathrm{F}$ \\
\hline I. Derivadas de la labor educativa en el aula & $2.91 \mathrm{a}$ & 2.99 & $3.32 \mathrm{a}$ & \multicolumn{1}{c}{$4.46^{*}$} \\
II. Derivadas de la vida cotidiana en el aula & 3.11 & 3.19 & 3.31 & 1.03 \\
III. Organización administrativa & $3.30 \mathrm{a}$ & $3.32 \mathrm{~b}$ & $3.75 \mathrm{ab}$ & $7.02^{* *}$ \\
IV. Flexibilidad respecto a la tarea docente & 3.28 & 3.34 & 3.32 & 0.12 \\
Total & 3.15 & 3.21 & 3.42 & 2.41 \\
\hline
\end{tabular}

${ }^{*} p<.05{ }^{* *} p<.01$

Las diferencias por pares de grupos se realizaron a través del test de Scheffé: a, b, c. 
Satisfacción Laboral y Fuentes de Presión Laboral según categoría

La tabla 18 muestra que los docentes que tienen la categoría de principales obtienen mayores promedios estadísticamente significativos en los subfactores línea de carrera y seguridad en el puesto que los docentes de la categoría de asociados y auxiliares.
La tabla 19 muestra que no existen diferencias estadísticamente significativas entre los docentes de la categoría principal, asociado y auxiliar en el Inventario de Fuentes de Presión Laboral al que se enfrenta el docente.

Tabla 18

Análisis de la varianza de la comparación de la Satisfacción Laboral según categoría

\begin{tabular}{lcccc}
\hline Satisfacción laboral & $\begin{array}{c}\text { Principal } \\
(\mathrm{n}=107) \\
\text { Media }\end{array}$ & $\begin{array}{c}\text { Asociado } \\
(\mathrm{n}=198) \\
\text { Media. }\end{array}$ & $\begin{array}{c}\text { Auxiliar } \\
(\mathrm{n}=198) \\
\text { Media }\end{array}$ & $\mathrm{F}$ \\
\hline Intrínseca & 4.36 & 4.28 & 4.26 & 0.93 \\
Extrínseca & 4.04 & 4.00 & 3.92 & 1.49 \\
Total & 4.19 & 4.13 & 4.07 & 1.24 \\
Independencia & 4.85 & 4.82 & 4.91 & 0.69 \\
Variedad & 4.22 & 4.30 & 4.28 & 0.35 \\
Libertad de cátedra & 4.58 & 4.44 & 4.49 & 1.19 \\
Línea de carrera & $4.17 \mathrm{a}$ & 4.05 & $3.88 \mathrm{a}$ & $3.49^{*}$ \\
Reconocimiento y distinción & 4.00 & 3.80 & 3.73 & 2.44 \\
Compensación económica & 3.54 & 3.45 & 3.30 & 2.25 \\
Condiciones de trabajo & 4.09 & 4.08 & 4.06 & 0.12 \\
Seguridad en el puesto & $4.02 \mathrm{a}$ & $3.98 \mathrm{~b}$ & $3.73 \mathrm{a} \mathrm{b}$ & $8.80^{* * *}$ \\
Estatus & 4.74 & 4.69 & 4.67 & 0.24 \\
Calidad en las relaciones interpersonales & 4.08 & 3.98 & 3.95 & 0.81 \\
Capacitación y perfeccionamiento & 3.76 & 3.78 & 3.80 & 0.51 \\
\hline
\end{tabular}

${ }^{*} p<.05{ }^{* * *} p<.001$

Las diferencias por pares de grupos se realizaron a través del test de Scheffé: a, b, c.

La diferencia entre las medias es significativa al nivel .05.

Tabla 19

Análisis de la varianza de la comparación de la Presión Laboral según categoría

\begin{tabular}{lcccc}
\hline Presión laboral & $\begin{array}{c}\text { Principal } \\
\mathrm{n}=107 \\
\text { Media }\end{array}$ & $\begin{array}{c}\text { Asociado } \\
\mathrm{n}=198 \\
\text { Media }\end{array}$ & $\begin{array}{c}\text { Auxiliar } \\
\mathrm{n}=198 \\
\text { Media }\end{array}$ & $\mathrm{F}$ \\
\hline I. Derivadas de la labor educativa en el aula & 2.97 & 2.99 & 3.06 & 0.37 \\
II. Derivadas de la vida educativa cotidiana en el aula & 3.19 & 3.14 & 3.27 & 0.42 \\
III. Organización administrativa & 3.42 & 3.35 & 3.40 & 0.28 \\
IV. Flexibilidad respecto a la tarea docente & 3.35 & 3.33 & 3.30 & 0.71 \\
Total & 3.23 & 3.20 & 3.25 & 0.14 \\
\hline
\end{tabular}


Satisfacción Laboral y Fuentes de Presión Laboral según el ejercicio de la docencia en una sola universidad y en varias universidades

La tabla 20 muestra que los docentes que laboran en una sola universidad obtienen mayores promedios estadísticamente significativos en todos los subfactores de la Escala de Satisfacción Laboral, excepto en los subfactores Independencia y Seguridad en el puesto, que aquellos que trabajan en varias universidades.
La tabla 21 muestra que los docentes que laboran en más de una universidad obtienen mayores promedios en los factores de la escala de Fuentes de Presión Laboral que los docentes que trabajan en una sola universidad. Al realizarse las comparaciones se encuentran diferencias estadísticamente significativas en todos los factores, excepto en el factor Presiones sociales derivadas de la labor educativa en el aula.

Tabla 20

Prueba $Z$ de la diferencia de medias en el análisis comparativo de docentes según laboren en otra universidad

\begin{tabular}{lrrrrr}
\hline \multirow{2}{*}{ Satisfacción laboral } & \multicolumn{2}{c}{$\begin{array}{c}\text { Sí trabaja } \\
\mathrm{n}=195\end{array}$} & \multicolumn{2}{c}{ No trabaja } \\
& Media & D.E. & Media & D.E. & \\
& 4.18 & 0.65 & 4.37 & 0.65 & $-3.25^{* *}$ \\
Intrínseca & 3.86 & 0.61 & 4.05 & 0.63 & $-3.34^{* *}$ \\
Extrínseca & 4.00 & 0.60 & 4.20 & 0.61 & $-3.46^{* *}$ \\
Total & 4.87 & 0.73 & 4.89 & 0.75 & -0.20 \\
Independencia & 4.15 & 0.75 & 4.37 & 0.77 & $-3.12^{* *}$ \\
Variedad & 4.39 & 0.71 & 4.57 & 0.76 & $-2.68^{* *}$ \\
Libertad de cátedra & 3.82 & 1.00 & 4.12 & 0.91 & $-3.41^{* *}$ \\
Línea de carrera & 3.65 & 1.11 & 3.92 & 0.98 & $-2.81^{* *}$ \\
Reconocimiento y distinciones & 3.21 & 0.91 & 3.52 & 1.02 & $-3.52^{* *}$ \\
Compensación económica & 3.97 & 0.67 & 4.14 & 0.73 & $-2.61^{* *}$ \\
Condiciones de trabajo & 3.86 & 0.73 & 3.91 & 0.71 & -0.71 \\
Seguridad en el puesto & 4.60 & 0.81 & 4.78 & 0.76 & $-2.57^{*}$ \\
Estatus & 3.85 & 0.91 & 4.09 & 0.89 & $-2.92^{* *}$ \\
Calidad de las relaciones interpersonales & 3.66 & 1.00 & 3.85 & 1.03 & $-2.05^{*}$ \\
Capacitación y perfeccionamiento & & & & &
\end{tabular}

${ }^{*} p<.05{ }^{* *} p<.01$

Tabla 21

Prueba $Z$ de diferencia de medias en el análisis comparativo de la Presión laboral según laboren en otra universidad

\begin{tabular}{|c|c|c|c|c|c|}
\hline \multirow[t]{2}{*}{ Presión laboral } & \multicolumn{2}{|c|}{$\begin{array}{c}\text { Nombrado } \\
\mathrm{n}=195\end{array}$} & \multicolumn{2}{|c|}{$\begin{array}{c}\text { Contratado } \\
n=299\end{array}$} & \multirow[t]{2}{*}{ z } \\
\hline & Media & D.E. & Media & D.E. & \\
\hline I. Derivadas de la labor educativa en el aula & 3.13 & 1.00 & 2.95 & 1.01 & 1.89 \\
\hline II. Derivadas de la vida educativa cotidiana en el aula & 3.34 & 1.00 & 3.09 & 0.97 & $2.83^{* *}$ \\
\hline III. Organización administrativa & 3.50 & 0.90 & 3.33 & 0.93 & $2.05^{*}$ \\
\hline IV. Flexibilidad respecto a la tarea docente & 3.47 & 1.26 & 3.24 & 1.16 & $2.03^{*}$ \\
\hline Total & 3.36 & 0.93 & 3.15 & 0.87 & $2.50^{*}$ \\
\hline
\end{tabular}

${ }^{*} p<.05^{* *} p<.01$ 
Satisfacción Laboral y Fuentes de Presión Laboral según su formación

La tabla 22 muestra que los docentes que se formaron en universidades privadas obtienen mayores promedios estadísticamente significativos en todos los factores y subfactores de la Escala de Satisfacción Laboral, excepto en los subfactores Seguridad en el Puesto y
Estatus que los docentes que estudiaron en las universidades estatales.

La tabla 23 muestra que los docentes que se formaron en universidades estatales obtienen mayores promedios estadísticamente significativos en todos los factores de la Escala de Presión Laboral que los docentes que estudiaron en las universidades privadas.

\section{Tabla 22}

Prueba $Z$ de la diferencia de medias en la comparación de la satisfacción laboral según formación académica

\begin{tabular}{|c|c|c|c|c|c|}
\hline \multirow{2}{*}{ Satisfacción laboral } & \multicolumn{2}{|c|}{$\begin{array}{c}\text { Sí trabaja } \\
n=195\end{array}$} & \multicolumn{2}{|c|}{$\begin{array}{c}\text { No trabaja } \\
n=299\end{array}$} & \multirow[t]{2}{*}{ Z } \\
\hline & Media & D.E. & Media & D.E. & \\
\hline Intrínseca & 4,16 & 0.63 & 4.52 & 0.63 & $-6.14^{* * *}$ \\
\hline Extrínseca & 3.82 & 0.57 & 4.22 & 0.63 & $-7.11^{* * *}$ \\
\hline Total & 3.98 & 0.57 & 4.36 & 0.60 & $-7.01^{* * *}$ \\
\hline Independencia & 4.75 & 0.78 & 5.07 & 0.65 & $4.60^{* \star \star}$ \\
\hline Variedad & 4.17 & 0.75 & 4.46 & 0.74 & $-4.18^{\star \star *}$ \\
\hline Libertad de cátedra & 4.39 & 0.76 & 4.65 & 0.69 & $-3.92^{* * *}$ \\
\hline Línea de carrera & 3.87 & 0.92 & 4.26 & 0.93 & $-4.54^{* * *}$ \\
\hline Reconocimiento y distinciones & 3.62 & 0.99 & 4.16 & 1.00 & $-5.84^{\star \star *}$ \\
\hline Compensación económica & 3.22 & 0.96 & 3.73 & 0.94 & $-5.73^{* * *}$ \\
\hline Condiciones de trabajo & 3.92 & 0.64 & 4.34 & 0.74 & $-6.69^{* * *}$ \\
\hline Seguridad en el puesto & 3.84 & 0.68 & 3.96 & 0.77 & -1.86 \\
\hline Estatus & 4.63 & 0.80 & 4.80 & 0.76 & -2.35 \\
\hline Calidad de las relaciones interpersonales & 3.82 & 0.89 & 4.29 & 0.84 & $-5.63^{\star \star *}$ \\
\hline Capacitación y perfeccionamiento & 3.53 & 0.98 & 4.21 & 0.95 & $-7.69^{\star \star *}$ \\
\hline
\end{tabular}

$* * \star p<.001$

Tabla 23

Prueba $\mathbf{Z}$ de diferencia de medias de la comparación de la presión laboral según la formación académica

\begin{tabular}{lccccc}
\hline Presión laboral & \multicolumn{3}{c}{ Univ. estatal } & \multicolumn{3}{c}{ Univ. privada } & Z \\
& Media $=315$ & D.E. & Media & D.E. & \\
\hline I. Derivadas de la labor educativa en el aula & 3.17 & 0.95 & 2.75 & 1.05 & $4.51^{* *}$ \\
II. Derivadas de la vida cotidiana en el aula & 3.27 & 1.00 & 3.05 & 0.96 & $2.44^{*}$ \\
III. Organización administrativa & 3.52 & 0.88 & 3.15 & 0.95 & $4.42^{* * *}$ \\
IV. Flexibilidad respecto a la tarea docente & 3.41 & 1.20 & 3.19 & 1.21 & $1.96^{*}$ \\
Total & 3.34 & 0.89 & 3.04 & 0.90 & $3.71^{* * *}$ \\
\hline
\end{tabular}

${ }^{*} p<.05{ }^{* *} p<.01{ }^{* * *} p<.001$ 
Satisfacción Laboral y Fuentes de Presión Laboral según el estado civil

La tabla 24 muestra que los docentes de estado civil casados obtienen mayores promedios estadísticamente significativos en el subfactor Seguridad en el puesto que los solteros.
La tabla 25 muestra que los docentes casados obtienen mayores promedios estadísticamente significativos en todos los factores de la Escala de Fuentes de Presión Laboral que los docentes solteros. Al realizarse las comparaciones se encuentran diferencias estadísticamente significativas en todos los factores.

Tabla 24

Análisis comparativo de la satisfacción laboral según estado civil

\begin{tabular}{lccccc}
\hline \multirow{2}{*}{ Satisfacción laboral } & \multicolumn{2}{c}{$\begin{array}{c}\text { Soltero } \\
\mathrm{n}=199\end{array}$} & \multicolumn{2}{c}{$\begin{array}{c}\text { Casado } \\
\mathrm{n}=247\end{array}$} & $\mathrm{Z}$ \\
& Media & D.E. & Media & D.E. & \\
\hline SuBfACTORES & & & & & \\
Independencia & 4.94 & 0.70 & 4.86 & 0.74 & 1.17 \\
Variedad & 4.28 & 0.77 & 4.29 & 0.72 & -0.07 \\
Libertad de cátedra & 4.48 & 0.73 & 4.51 & 0.71 & -0.43 \\
Línea de carrera & 3.92 & 0.95 & 4.07 & 0.96 & -1.70 \\
Reconocimiento y distinciones & 3.78 & 1.06 & 3.84 & 1.01 & -0.64 \\
Compensación económica & 3.43 & 0.98 & 3.41 & 1.01 & 0.25 \\
Condiciones de trabajo & 4.07 & 0.75 & 4.08 & 0.65 & -0.16 \\
Seguridad en el puesto & 3.79 & 0.70 & 3.94 & 0.71 & $-2.14^{*}$ \\
Estatus & 4.65 & 0.79 & 4.73 & 0.78 & -1.04 \\
Calidad de relaciones interpersonales & 3.97 & 0.91 & 4.02 & 0.91 & -0.57 \\
Capacitación y perfeccionamiento & 3.78 & 1.00 & 3.79 & 1.02 & -0.11 \\
FACTORES & & & & & \\
Intrínseca & 4.28 & 0.65 & 4.31 & 0.64 & -0.56 \\
Extrínseca & 3.95 & 0.63 & 3.99 & 0.62 & -0.75 \\
Total Satisfacción Laboral & 4.10 & 0.61 & 4.14 & 0.60 & -0.69 \\
\hline
\end{tabular}

${ }^{*} p<.05$

Tabla 25

Prueba Z de diferencia de medias de la comparación de la presión laboral de acuerdo con el estado civil

\begin{tabular}{lccccc}
\hline \multirow{2}{*}{ Presión laboral } & \multicolumn{2}{c}{$\begin{array}{c}\text { Soltero } \\
n=199\end{array}$} & \multicolumn{3}{c}{$\begin{array}{c}\text { Casado } \\
n=247\end{array}$} \\
& Media & D.E. & Media & D.E. \\
\hline I. Derivadas de la labor educativa en el aula & 2.90 & 1.02 & 3.09 & 1.01 & $-1.97^{*}$ \\
II. Derivadas de la vida cotidiana en el aula & 3.07 & 0.97 & 3.27 & 1.01 & $-2.15^{*}$ \\
III. Organización administrativa & 3.27 & 0.94 & 3.47 & 0.91 & $-2.25^{*}$ \\
IV. Flexibilidad respecto a la tarea docente & 3.21 & 1.16 & 3.44 & 1.28 & -1.93 \\
Total & 3.11 & 0.89 & 3.32 & 0.93 & $-2.35^{*}$ \\
\hline
\end{tabular}

$* p<.05$ 
Satisfacción Laboral y Fuentes de Presión Laboral según sexo

La tabla 26 muestra que no se encuentran diferencias estadísticamente significativas en ninguno de los factores y subfactores entre docentes de sexo masculino y docentes de sexo femeni- no en la Escala de Satisfacción Laboral.

La tabla 27 muestra que no se encuentran diferencias estadísticamente significativas en los diferentes factores del Inventario de Fuentes de Presión Laboral entre los docentes según sexo.

Tabla 26

Prueba $Z$ de diferencia de medias en el análisis comparativo de la Satisfacción Laboral según sexo

\begin{tabular}{lccccc}
\hline \multirow{2}{*}{ Satisfacción laboral } & \multicolumn{2}{c}{$\begin{array}{c}\text { Masculino } \\
\mathrm{n}=375\end{array}$} & \multicolumn{2}{c}{$\begin{array}{c}\text { Femenino } \\
\mathrm{n}=129\end{array}$} & $\mathrm{Z}$ \\
& Media & D.E. & Media & D.E. & \\
\hline Intrínseca & 4.31 & 0.68 & 4.22 & 0.59 & 1.35 \\
Extrínseca & 3.99 & 0.64 & 3.91 & 0.60 & 1.23 \\
Total & 4.14 & 0.63 & 4.05 & 0.56 & 1.35 \\
Independencia & 4.88 & 0.75 & 4.83 & 0.75 & 0.53 \\
Variedad & 4.29 & 0.79 & 4.23 & 0.69 & 0.81 \\
Libertad de cátedra & 4.52 & 0.74 & 4.38 & 0.73 & 1.88 \\
Línea de carrera & 4.03 & 0.96 & 3.94 & 0.90 & 0.96 \\
Reconocimiento y distinciones & 3.84 & 1.05 & 3.73 & 0.97 & 1.09 \\
Compensación económica & 3.41 & 1.03 & 3.39 & 0.86 & 0.17 \\
Condiciones de trabajo & 4.08 & 0.70 & 4.05 & 0.73 & 0.50 \\
Seguridad en el puesto & 3.92 & 0.73 & 3.79 & 0.68 & 1.77 \\
Estatus & 4.73 & 0.77 & 4.60 & 0.83 & 1.61 \\
Calidad de relaciones interpersonales & 4.01 & 0.92 & 3.91 & 0.84 & 1.12 \\
Capacitación y perfeccionamiento & 3.80 & 1.03 & 3.74 & 0.98 & 0.57 \\
\hline
\end{tabular}

Tabla 27

Prueba $Z$ de diferencia de medias del análisis comparativo de Presión Laboral según sexo

\begin{tabular}{lccccr}
\hline Presión laboral & \multicolumn{2}{c}{$\begin{array}{c}\text { Masculino } \\
n=375\end{array}$} & \multicolumn{2}{c}{$\begin{array}{c}\text { Femenino } \\
n=129\end{array}$} & Z \\
& Media & D.E. & Media & D.E. \\
\hline I. Derivadas de la labor educativa en el aula & 3.03 & 1.01 & 2.98 & 1.01 & 0.47 \\
II. Derivadas de la vida cotidiana en el aula & 3.17 & 1.00 & 3.21 & 0.98 & -0.39 \\
III. Organización administrativa & 3.36 & 0.93 & 3.35 & 0.91 & -0.87 \\
IV. Flexibilidad respecto de la tarea docente & 3.32 & 1.23 & 3.34 & 1.16 & -0.18 \\
Total & 3.22 & 0.92 & 3.24 & 0.89 & -0.26 \\
\hline
\end{tabular}


Satisfacción Laboral y Fuentes de

Presión Laboral según lugar de nacimiento

La tabla 28 muestra que no se encuentran diferencias estadísticamente significativas en los factores y subfactores en la Escala de satisfacción laboral entre los docentes según lugar de nacimiento.

La tabla 29 muestra que no se encuentran diferencias estadísticamente significativas en los factores del inventario de presión a los que se enfrenta el docente según lugar de nacimiento.

Tabla 28

Prueba $Z$ de diferencia de medias en la comparación de la Satisfacción Laboral según lugar de nacimiento

\begin{tabular}{lrrrrr}
\hline \multirow{2}{*}{ Satisfacción laboral } & \multicolumn{2}{c}{ Lima } & \multicolumn{2}{c}{$\begin{array}{c}\text { Provincias } \\
\mathrm{n}=322\end{array}$} \\
& Media & D.E. & Media & D.E. & \\
\hline Intrínseca & 4.29 & 0.66 & 429 & 0.66 & 0.09 \\
Extrínseca & 3.96 & 0.64 & 3.99 & 0.60 & -0.40 \\
Total & 4.11 & 0.62 & 4.12 & 0.59 & -0.18 \\
Independencia & 4.91 & 0.71 & 4.78 & 0.82 & 1.92 \\
Variedad & 4.23 & 0.76 & 4.36 & 0.76 & -1.69 \\
Libertad de cátedra & 4.46 & 0.73 & 4.54 & 0.0 .76 & -1.20 \\
Línea de carrera & 4.03 & 0.94 & 3.97 & 0.97 & 0.75 \\
Reconocimiento y distinciones & 3.83 & 1.05 & 3.80 & 1.00 & 0.32 \\
Compensación económica & 3.44 & 0.98 & 3.35 & 1.00 & 1.06 \\
Condiciones de trabajo & 4.05 & 0.76 & 4.11 & 0.60 & -0.95 \\
Seguridad en el puesto & 3.85 & 0.72 & 3.95 & 0.70 & -1.44 \\
Estatus & 4.64 & 0.79 & 4.78 & 0.77 & -1.90 \\
Calidad de relaciones interpersonales & 3.99 & 0.90 & 3.99 & 0.91 & 0.54 \\
Capacitación y perfeccionamiento & 3.80 & 1.00 & 3.74 & 1.05 & 0.59 \\
\hline
\end{tabular}

Tabla 29

Prueba $Z$ de la diferencia de media de la comparación de la Presión Laboral según lugar de nacimiento

\begin{tabular}{lcccrr}
\hline & \multicolumn{3}{c}{$\begin{array}{c}\text { Masculino } \\
\text { Presión laboral }\end{array}$} & \multicolumn{3}{c}{$\begin{array}{c}\text { Femenino } \\
n=322\end{array}$} & Z \\
& Media & D.E. & Media & D.E. \\
\hline I. Derivadas de la labor educativa en el aula & 3.01 & 1.02 & 3.01 & 0.99 & -0.13 \\
II. Derivadas de la vida cotidiana en el aula & 3.19 & 1.00 & 3.16 & 0.99 & 0.37 \\
III. Organización administrativa & 3.37 & 0.94 & 3.39 & 0.90 & -0.24 \\
IV. Flexibilidad respecto a la tarea docente & 3.31 & 1.19 & 3.33 & 1.24 & -0.23 \\
Total & 3.22 & 0.91 & 3.22 & 0.90 & -0.07 \\
\hline
\end{tabular}


Satisfacción Laboral y Fuentes de Presión Laboral según tiempo de servicios

Este análisis busca identificar la variable que actúa como factor al obtener las correlaciones entre satisfacción, presión laboral y tiempo de servicio docente y años de servicio en la universidad. Igualmente, se realiza el análisis por conglomerados, que permite identificar la existencia de perfiles de los docentes de acuerdo con su nivel de Satisfacción Laboral y de Presión Laboral.
Correlación de Satisfacción Laboral y tiempo de servicio en la universidad y en la docencia

La tabla 30 señala que los años de trabajo en la docencia presentan correlación significativa pero baja con el subfactor Seguridad en el puesto. En lo que concierne a los años de trabajo en la universidad, también se encuentran correlaciones significativas negativas y bajas en el caso de Satisfacción Intrínseca, Satisfacción Total, Independencia, Reconocimiento y distinciones, Compensación económica, Condiciones de trabajo, Seguridad en el puesto y Capacitación y perfeccionamiento.

Tabla 30

Matriz de correlaciones de la Satisfacción Laboral con los años de servicio en la docencia y el trabajo en la universidad

\begin{tabular}{lcc}
\hline Satisfacción laboral & $\begin{array}{c}\text { Años de trabajo } \\
\text { en docencia }\end{array}$ & $\begin{array}{c}\text { Años de trabajo } \\
\text { en la universidad }\end{array}$ \\
\hline Intrínseca & -0.01 & $-0.09^{*}$ \\
Extrínseca & -0.01 & -0.09 \\
Total & -0.01 & $-0.09^{*}$ \\
Independencia & -0.029 & $-0.12^{*}$ \\
Variedad & -0.01 & -0.08 \\
Libertad de cátedra & -0.01 & -0.06 \\
Línea de carrera & 0.03 & -0.01 \\
Reconocimiento y distinciones & -0.02 & $-0.10^{*}$ \\
Compensación económica & -0.03 & $-0.12^{* *}$ \\
Condiciones de trabajo & -0.05 & $-0.13^{\star *}$ \\
Seguridad en el puesto & $0.11^{*}$ & $0.11^{*}$ \\
Estatus & 0.06 & 0.01 \\
Calidad de relaciones interpersonales & -0.02 & -0.06 \\
Capacitación y perfeccionamiento & -0.07 & $-0.14^{* *}$ \\
\hline
\end{tabular}

${ }^{*} p<.05 \quad$ ** $p<.01$ 
En el caso de la correlación de los factores de las Fuentes de Presión Laboral y los años de servicio y los factores de las Fuentes de Presión Laboral y los años de trabajo en la universidad (tabla 31) no se encuentran correlaciones significativas.

\section{Identificación de perfiles entre los docentes}

El análisis de conglomerados se realizó con el objetivo de observar si existen agrupamientos de sujetos con carac- terísticas similares. Los resultados presentados en la tabla 32 indican que según el tipo de Presión Laboral se puede identificar la existencia de tres grupos de docentes: con Alta, Media y Baja Presión Laboral.

El Conglomerado 1 está conformado por 147 casos $(29,05 \%)$ e incluye a los docentes que presentan los puntajes más bajos en los factores de la Escala de Fuentes de Presión Laboral.

El Conglomerado 2 está conformado por 200 casos $(39,52 \%)$ e incluye a

Tabla 31

Matriz de correlaciones de la Presión Laboral con los años de servicio en la docencia y el trabajo en la universidad

\begin{tabular}{lcc}
\hline Presión laboral & $\begin{array}{c}\text { Años de trabajo } \\
\text { en docencia }\end{array}$ & $\begin{array}{c}\text { Años de trabajo } \\
\text { en la universidad }\end{array}$ \\
\hline I. Derivadas de la labor educativa en el aula & .022 & .001 \\
II. Derivadas de la vida cotidiana en el aula & -.020 & -.044 \\
III. Organización administrativa & .049 & .031 \\
IV. Flexibilidad respecto a la tarea docente & -.033 & -.067 \\
Total & .002 & -0.26 \\
\hline
\end{tabular}

Tabla 32

Tabla de análisis descriptivos de los conglomerados de Presión Laboral

\begin{tabular}{|c|c|c|c|c|c|c|c|c|}
\hline \multirow[t]{2}{*}{ Fuentes de presión laboral } & \multicolumn{2}{|c|}{$\begin{array}{c}\text { Bajo } \\
N=147\end{array}$} & \multicolumn{2}{|c|}{$\begin{array}{l}\text { Medio } \\
N=200\end{array}$} & \multicolumn{2}{|c|}{$\begin{array}{c}\text { Alto } \\
N=159\end{array}$} & \multicolumn{2}{|c|}{$\begin{array}{c}\text { Total } \\
\mathrm{N}=506\end{array}$} \\
\hline & M & D.E & M. & D.E. & M. & D.E. & M. & D.E \\
\hline $\begin{array}{l}\text { I. Derivadas de la labor educativa } \\
\text { en el aula }\end{array}$ & 1.88 & 0.55 & 3.05 & 0.64 & 4.00 & 0.53 & 3.01 & 1.01 \\
\hline $\begin{array}{l}\text { II. Derivadas de la vida cotidiana } \\
\text { en el aula }\end{array}$ & 2.10 & 0.55 & 3.19 & 0.57 & 4.18 & 0.60 & 3.18 & 0.99 \\
\hline III. Organización administrativa & 2.34 & 0.66 & 3.47 & 0.55 & 4.23 & 0.48 & 3.38 & 0.93 \\
\hline $\begin{array}{l}\text { IV. Flexibilidad respecto a la tarea } \\
\text { docente }\end{array}$ & 2.07 & 0.86 & 3.32 & 0.74 & 4.48 & 0.72 & 3.32 & 1.21 \\
\hline Total & 2.10 & 0.43 & 3.26 & 0.33 & 4.22 & 0.38 & 3.22 & 0.91 \\
\hline
\end{tabular}


los docentes que presentan los puntajes intermedios en todos los factores de la Escala de Fuentes de Presión Laboral.

El Conglomerado 3 está conformado por 159 casos $(31,42 \%)$ e incluye a los docentes que presentan los puntajes más altos en todos los factores de la Escala de Fuentes de Presión Laboral.

De manera similar, en la Satisfacción Laboral se identifican tres subgrupos: Alto, Medio y Bajo (véase tabla 33).

De esta manera, el Conglomerado 1 está conformado por 136 casos $(26,87 \%)$ e incluye a los docentes que presentan los puntajes más bajos en los factores de la Escala de Satisfacción Laboral. El Conglomerado 2 está con- formado por 240 casos $(47,43 \%)$ e incluye a los docentes que presentan los puntajes intermedios en todos los factores de la Escala de Satisfacción Laboral. El Conglomerado 3 está conformado por 130 casos $(25,69 \%)$ e incluye a los docentes que presentan los puntajes más altos en todos los factores de la Escala de Satisfacción Laboral.

El análisis de tabulación cruzada de los conglomerados de Presión Laboral identificados según tipo de gestión de la universidad presentado en la tabla 34 indica que sí existen diferencias estadísticamente significativas (Chi cuadrado $=10.96$ g.l. $=2 \mathrm{p}=0.004)$, por lo que se puede concluir que los conglomerados se distribuyen de

Tabla 33

Tabla de análisis descriptivos de los conglomerados de Satisfacción Laboral

\begin{tabular}{lccccccccc}
\hline & \multicolumn{2}{c}{ Bajo } & \multicolumn{2}{c}{ Intermedio } & \multicolumn{2}{c}{ Alto } & \multicolumn{2}{c}{ Total } \\
& \multicolumn{2}{c}{$\mathrm{N}=136$} & \multicolumn{2}{c}{$\mathrm{N}=240$} & \multicolumn{2}{c}{$\mathrm{N}=130$} & \multicolumn{2}{c}{$\mathrm{N}=506$} \\
& $\mathrm{M}$ & $\mathrm{D} . \mathrm{E}$ & $\mathrm{M}$. & $\mathrm{D} . \mathrm{E}$. & $\mathrm{M}$. & $\mathrm{D} . \mathrm{E}$. & $\mathrm{M}$. & $\mathrm{D} . \mathrm{E}$ \\
\hline Independencia & 4.75 & 0.68 & 4.62 & 0.73 & 5.44 & 0.52 & 4.87 & 0.75 \\
Variedad & 3.69 & 0.62 & 4.19 & 0.56 & 5.04 & 0.59 & 4.27 & 0.77 \\
Libertad de cátedra & 4.23 & 0.67 & 4.27 & 0.65 & 5.14 & 0.57 & 4.48 & 0.74 \\
Línea de carrera & 2.99 & 0.69 & 4.07 & 0.53 & 4.96 & 0.69 & 4.01 & 0.95 \\
Reconocimiento y distinción & 2.65 & 0.70 & 3.84 & 0.51 & 4.97 & 0.63 & 3.81 & 1.03 \\
Compensación económica & 2.53 & 0.58 & 3.38 & 0.69 & 4.37 & 0.90 & 3.41 & 0.99 \\
Condiciones de trabajo & 3.60 & 0.61 & 3.94 & 0.50 & 4.82 & 0.53 & 4.07 & 0.71 \\
Seguridad en el puesto & 3.54 & 0.68 & 3.78 & 0.55 & 4.43 & 0.73 & 3.88 & 0.72 \\
Estatus & 4.40 & 0.76 & 4.47 & 0.68 & 5.41 & 0.50 & 4.69 & 0.79 \\
Calidad de relaciones interpersonales & 3.23 & 0.76 & 3.92 & 0.60 & 4.92 & 0.64 & 3.99 & 0.90 \\
Capacitación y perfeccionamiento & 2.78 & 0.67 & 3.78 & 0.66 & 4.82 & 0.79 & 3.78 & 1.02 \\
Intrínseca & 3.66 & 0.36 & 4.20 & 0.38 & 5.11 & 0.41 & 4.29 & 0.66 \\
Extrínseca & 3.35 & 0.31 & 3.88 & 0.30 & 4.79 & 0.40 & 3.97 & 0.63 \\
Total & 3.49 & 0.27 & 4.02 & 0.28 & 4.94 & 0.36 & 4.12 & 0.61 \\
\hline
\end{tabular}


Tabla 34

Tabla de contingencia del conglomerado Presión Laboral según el tipo de gestión de la universidad

\begin{tabular}{lcccc}
\hline Gestión & Alto & Medio & Bajo & Total \\
\hline Estatal & $\mathbf{7 9}$ & $\mathbf{8 7}$ & 46 & 212 \\
& $\mathbf{1 5 . 6 \%}$ & $\mathbf{1 7 . 2 \%}$ & $9.1 \%$ & $41.9 \%$ \\
Particular & 80 & $\mathbf{1 1 3}$ & $\mathbf{1 0 1}$ & 294 \\
Total & $15.8 \%$ & $\mathbf{2 2 . 3 \%}$ & $\mathbf{2 0 \%}$ & $58.1 \%$ \\
& 159 & 200 & 147 & 506 \\
& $31.4 \%$ & $39.5 \%$ & $29.1 \%$ & $100 \%$ \\
\hline
\end{tabular}

Chi cuadrado $=10.96 \quad$ g.l. $=2 \quad p=0.004$

Tabla 35

Tabla de contingencia del conglomerado Satisfacción Laboral según el tipo de gestión de la universidad

\begin{tabular}{lcccc}
\hline Gestión & Alto & Medio & Bajo & Total \\
\hline Estatal & 13 & 100 & 99 & 212 \\
& $2.6 \%$ & $19.7 \%$ & $19.6 \%$ & $41.9 \%$ \\
Particular & 117 & 140 & 37 & 294 \\
Total & $\mathbf{2 3 . 1 \%}$ & $\mathbf{2 7 . 7 \%}$ & $7.3 \%$ & $58.1 \%$ \\
& 130 & 240 & 136 & 506 \\
& $25.7 \%$ & $47.4 \%$ & 26.9 & $100 \%$ \\
\hline
\end{tabular}

Chi cuadrado $=107.67 \quad$ g.l. $=2 \quad p=0.0001$

forma diferenciada en los tipos de gestión de la universidad. En el grupo estatal prevalecen los conglomerados altos y medios $(32,8 \%)$ y en el grupo de docentes de universidades privadas los conglomerados medios y bajos $(42,3 \%)$.

En relación con el análisis de tabulación cruzada de los conglomerados de Satisfacción Laboral identificados según tipo de gestión de la universidad presentado en la tabla 35, apreciamos que la existencia de diferencias estadísticamente significativas (Chi cuadrado
$=107.67 \mathrm{gl}=2 \mathrm{p}=0.0001$ ), por lo que se puede concluir que los conglomerados se distribuyen de forma diferenciada en los tipos de gestión de la universidad. En el grupo de docentes estatales predominan los conglomerados medios y bajos $(39,3 \%)$ y en los docentes de universidades privadas los conglomerados altos y medios $(50,8 \%)$.

La relación entre las variables Satisfacción Laboral y Fuentes de Presión Laboral para el tipo de gestión estatal de universidad permite apreciar, 
según la tabla 36 , que la existencia de diferencias estadísticamente significativas $($ Chi cuadrado $=20.23 \mathrm{gl}=4$ $\mathrm{p}=0.0001$ ), por lo que se puede concluir que los conglomerados se distribuyen en forma diferenciada, notándose que los docentes que tienen alta Presión Laboral presentan media y baja Satisfacción y los que presentan Presión media y baja tienen baja Satisfacción.

La relación entre las variables Satisfacción Laboral y Fuentes de Presión Laboral, considerando el tipo de gestión particular de universidad, permite apreciar, según la tabla 37 , la existencia de diferencias estadísticamente significativas (Chi cuadrado $=11.54$ $\mathrm{gl}=4 \mathrm{p}=0.021$ ). Por lo tanto, se puede concluir que los conglomerados se distribuyen en forma diferenciada, así los docentes que tienen alta Presión Laboral muestran Satisfacción Laboral media y alta; en tanto que los docentes que presentan Presión Laboral media muestran Satisfacción Laboral alta y media y aquellos que presentan baja Presión Laboral muestran alta y media Satisfacción Laboral.

Tabla 36

Tabla de contingencia del conglomerado de Presión Laboral con el conglomerado Presión Laboral según el tipo de gestión estatal de la universidad

\begin{tabular}{cccccc}
\hline \multicolumn{5}{c}{ Conglomerado Satisfacción Laboral } \\
\cline { 2 - 6 } & C. presión & Alto & Medio & Bajo & Total \\
\hline \multirow{4}{*}{ Estatal } & Alto & 1 & $\mathbf{2 6}$ & $\mathbf{5 2}$ & 79 \\
& & $0.5 \%$ & $\mathbf{1 2 . 3} \%$ & $\mathbf{2 4 . 5} \%$ & $37.3 \%$ \\
& Medio & 8 & $\mathbf{4 9}$ & $\mathbf{3 0}$ & 87 \\
& & $3.8 \%$ & $\mathbf{2 3 . 1} \%$ & $\mathbf{1 4 . 1} \%$ & $41 \%$ \\
& Bajo & 4 & $\mathbf{2 5}$ & $\mathbf{1 7}$ & 46 \\
& & $1.9 \%$ & $\mathbf{1 1 . 8} \%$ & $\mathbf{8} \%$ & $21.7 \%$ \\
& Total & 13 & 100 & 99 & 212 \\
& & $6.1 \%$ & $47.2 \%$ & $46.7 \%$ & $100 \%$ \\
\hline
\end{tabular}

Chi cuadrado $=20.23 \quad$ g.l. $=4 \quad p=0.0001$

Tabla 37

Tabla de contingencia del conglomerado de presión laboral con el conglomerado presión laboral según el tipo de gestión particular de la universidad

\begin{tabular}{cccccc}
\hline \multicolumn{5}{c}{ Conglomerado Satisfacción Laboral } \\
\cline { 2 - 6 } & C. presión & Alto & Medio & Bajo & Total \\
\hline \multirow{4}{*}{ Estatal } & Alto & 33 & $\mathbf{3 3}$ & $\mathbf{1 4}$ & 80 \\
& & $11.2 \%$ & $\mathbf{1 1 . 2 \%}$ & $\mathbf{4 . 8 \%}$ & $27.2 \%$ \\
& Medio & $\mathbf{6 1}$ & $\mathbf{6 1}$ & 17 & 113 \\
& & $\mathbf{1 1 . 9 \%}$ & $\mathbf{2 0 . 7 \%}$ & $5.8 \%$ & $38.4 \%$ \\
& Bajo & $\mathbf{4 9}$ & $\mathbf{4 6}$ & 6 & 101 \\
& & $\mathbf{1 6 . 7 \%}$ & $\mathbf{1 5 . 6 \%}$ & $2.0 \%$ & $34.3 \%$ \\
& Total & 117 & 140 & 37 & 294 \\
& $39.8 \%$ & $47.4 \%$ & $12.6 \%$ & $99.9 \%$ \\
\hline
\end{tabular}

Chi cuadrado $=11.54$ g.l. $=4 \quad p=0.021$ 
Identificación de los componentes de Satisfacción Laboral y Fuentes de Presión Laboral con mayor y menor incidencia

Se analizaron los factores que muestran mayor incidencia, tanto en la Satisfacción Laboral como en la Presión Laboral, considerando a los docentes como un solo grupo y teniendo en cuenta el tipo de gestión.

En el Inventario de fuentes de presión a las que se enfrenta el docente, la muestra de docentes (tabla 38), el factor Presiones originadas por la organización administrativa alcanza el mayor promedio (3.38), y las fuentes deriva- das de la labor educativa en el aula alcanzan el menor promedio (3.01).

En cuanto a la Satisfacción Laboral (tabla 39), el factor Intrínseco alcanza el mayor promedio (4.29), y el Extrínseco el menor promedio (3.97). Esta situación se refleja cuando se analizan los subfactores, donde en los cuatro primeros lugares aparecen tres factores pertenecientes al factor Intrínseco (Independencia, Libertad de cátedra y Variedad, y en los dos últimos lugares figuran los subfactores considerados en el factor Extrínseco (Capacitación y perfeccionamiento y Compensación económica).

Tabla 38

Factores que muestran mayor incidencia en las Fuentes de Presión Laboral

\begin{tabular}{llc}
\hline Orden & \multicolumn{1}{c}{ Factor } & Promedio \\
\hline 1 & Originada por la organización administrativa & 3.38 \\
2 & Flexibilidad con respecto a la tarea docente & 3.32 \\
3 & Derivada de la vida cotidiana en el aula & 3.18 \\
4 & Derivada de la labor educativa en el aula & 3.01 \\
\hline
\end{tabular}

Tabla 39

Factores que muestran mayor incidencia en la Satisfacción Laboral

\begin{tabular}{llc}
\hline Orden & \multicolumn{1}{c}{ Factor } & Promedio \\
\hline 1 & Factor Intrínseco & 4.29 \\
2 & Factor Extrínseco & 3.97 \\
\hline 1 & Independencia & 4.87 \\
2 & Estatus & 4.69 \\
3 & Libertad de cátedra & 4.48 \\
4 & Variedad & 4.27 \\
5 & Condiciones de trabajo & 4.07 \\
6 & Línea de carrera & 4.01 \\
7 & Calidad de relaciones interpersonales & 3.99 \\
8 & Seguridad en el puesto & 3.88 \\
9 & Reconocimiento y distinciones & 3.81 \\
10 & Capacitación y perfeccionamiento & 3.78 \\
11 & Compensación económica & 3.41 \\
\hline
\end{tabular}


Considerando el tipo de gestión de la universidad, estatal y particular (tabla 40), se observa que en ambos tipos de gestión se mantiene el mismo orden en cuanto a las fuentes de presión a las que se ve sometido el docente universitario.

En cuanto a la Satisfacción Laboral (tabla 41), los docentes que laboran en universidades estatales obtienen los mayores promedios en los subfactores Independencia, Estatus, Libertad de cátedra y Variedad; en tanto que los menores promedios se obtienen en los subfactores Reconocimiento y distinciones, Capacitación y perfeccionamiento y Compensación económica.

De otro lado, los docentes que prestan servicios en las universidades particulares obtienen los mayores promedios en los subfactores Independencia, Estatus, Libertad de cátedra y Variedad; los menores promedios los obtienen en los subfactores Reconocimiento y distinciones, Seguridad en el puesto y Compensación económica.

Tabla 40

Factores que muestran mayor incidencia en las Fuentes de Presión Laboral según tipo de gestión de la universidad

\begin{tabular}{|c|c|c|c|}
\hline Universidad estatal & Promedio & Universidad particular & Promedio \\
\hline $\begin{array}{l}\text { Derivada de la labor educativa } \\
\text { en el aula }\end{array}$ & 3.65 & $\begin{array}{l}\text { Derivada de la labor educativa } \\
\text { en el aula }\end{array}$ & 3.25 \\
\hline Derivada de la vida cotidiana en el aula & 3.42 & Derivada de la vida cotidiana en el aula & 3.19 \\
\hline $\begin{array}{l}\text { Originada por la organización adminis- } \\
\text { trativa }\end{array}$ & 3.29 & $\begin{array}{l}\text { Originada por la organización adminis- } \\
\text { trativa }\end{array}$ & 3.11 \\
\hline $\begin{array}{l}\text { Flexibilidad con respecto a la tarea } \\
\text { docente }\end{array}$ & 3.29 & $\begin{array}{l}\text { Flexibilidad con respecto a la tarea } \\
\text { docente }\end{array}$ & 2.81 \\
\hline
\end{tabular}

Tabla 41

Factores que muestran mayor incidencia en la Satisfacción Laboral según tipo de gestión de la universidad

\begin{tabular}{lclc}
\hline \multicolumn{1}{c}{ Universidad estatal } & Promedio & \multicolumn{1}{c}{ Universidad particular } & Promedio \\
\hline Independencia & 4.71 & Independencia & 4.98 \\
Estatus & 4.56 & Estatus & 4.79 \\
Libertad de cátedra & 4.35 & Libertad de cátedra & 4.58 \\
Variedad & 4.05 & Variedad & 4.44 \\
Seguridad en el puesto & 3.81 & Condiciones de trabajo & 4.30 \\
Condiciones de trabajo & 3.76 & Calidad de relaciones interpersonales & 4.25 \\
Línea de carrera & 3.69 & Línea de carrera & 4.23 \\
Calidad de relaciones interpersonales & 3.62 & Capacitación y perfeccionamiento & 4.22 \\
Reconocimiento y distinciones & 3.34 & Reconocimiento y distinciones & 4.16 \\
Capacitación y perfeccionamiento & 3.16 & Seguridad en el puesto & 3.93 \\
Compensación económica & 2.77 & Compensación económica & 3.87 \\
\hline
\end{tabular}




\section{DISCUSIÓN}

La presente investigación tuvo como objetivo central conocer la relación existente entre las Fuentes de Presión Laboral y la Satisfacción Laboral entre los docentes universitarios de Lima metropolitana, considerando el tipo de gestión. Asimismo, se indagó la existencia de diferencias de la Satisfacción entre los docentes de las universidades estatales y privadas. Igualmente, analizó la posible existencia de diferencias de las Fuentes de Presión Laboral entre los docentes de las universidades estatales y privadas. Finalmente, se orientó a estudiar la influencia de las Fuentes de Presión Laboral sobre los Factores de la Satisfacción Laboral.

Una de las primeras tareas al abordar ese objetivo fue constatar la validez y confiabilidad de los instrumentos con que se iban a alcanzar los objetivos. Para tal fin se consideró como instrumentos de medición la Escala de Satisfacción Docente y el Cuestionario sobre Fuentes de Presión Laboral. El análisis factorial confirmatorio permitió afirmar que ambos instrumentos presentan validez de constructo, en razón de que se encuentran en forma consistente y adecuada en los datos obtenidos en la presente investigación. Los análisis de los ítems y la confiabilidad por consistencia interna de cada uno de los factores de ambos instrumentos, han permitido observar que en todos los casos las correlaciones ítem- test corregidas se encuentran dentro del criterio propuesto por Kline (1986), y en los coeficientes Alfa de Cronbach presentaron valores que pueden ser calificados como adecuados.

Podemos afirmar que la Escala de Satisfacción Laboral Docente y el Cuestionario sobre Fuentes de Presión Laboral presentan validez de constructo y confiabilidad por consistencia interna, con lo cual se cumple con los requerimientos psicométricos básicos para estos instrumentos, como lo proponen Martínez (1985), Marín (1986), Muñiz (1994, 1996) y Anastasi \& Urbina (1998).

Los análisis de bondad de ajuste a la curva normal realizados mediante la prueba de Kolmogorov-Smirnov (Siegel \& Castellán, 1995) permitieron conocer que los puntajes de los factores de la Escala de Satisfacción Docente y del Cuestionario sobre Fuentes de Presión Laboral presentaron distribuciones de puntajes aproximados a la distribución, lo que permitió conocer cómo se distribuyen en la muestra los instrumentos $\mathrm{y}$, por otra parte, aplicar análisis estadísticos más potentes con la finalidad de obtener resultados más precisos.

Los resultados obtenidos nos permiten afirmar que existe relación inversa entre las Fuentes de Presión Laboral y la Satisfacción Laboral de los docentes universitarios de Lima metropolitana. Así, se encontraron correlaciones negativas, que oscilan entre $-.11 \mathrm{y}-.35$, entre 
los factores Intrínseco y Extrínseco de la Escala de Satisfacción Laboral y los cuatro factores del Cuestionario de Fuentes de Presión Laboral. Asimismo, se encontraron correlaciones negativas en cada uno de los factores de las Fuentes de Presión Laboral y los diferentes subfactores de la Escala de Satisfacción Laboral; estas correlaciones son significativas en todos los casos, excepto en tres, entre el factor presiones originadas por la organización administrativa y el factor variedad, estatus y calidad de las relaciones interpersonales. Este hallazgo coincide con los de Cherniss (1992), quien sostiene la existencia de una correlación negativa entre Satisfacción Laboral síndrome del burnout.

En este contexto debemos indicar que la Presión Laboral sería un factor que atenta contra la Satisfacción Laboral del docente universitario en el desempeño de sus funciones. Las Fuentes de Presión Laboral deben ser entendidas como un conjunto de elementos provenientes de los factores intrínsecos del puesto o la tarea, de los roles que debe cumplir dentro de la organización, del desarrollo de su carrera profesional, de las relaciones interpersonales y las derivadas de la organización misma y de las condiciones físicas donde realiza el trabajo. En tanto, la Satisfacción Laboral es entendida como la actitud general del docente hacia su trabajo. El docente que tenga un alto grado de satisfacción mostrará actitudes positivas hacia el trabajo, mientras que el que está insatisfecho exhibirá actitudes negativas. La relación entre estas variables es significativa de manera consistente en la literatura especializada, ya sean estas variables estimadas de forma global o mediante sus diferentes dimensiones. Los valores de correlación entre ambas variables oscilan entre $\mathrm{r}=-.42 \mathrm{y}$ $r=-.64$ (Gil-Monte \& Peiró, 1997; Travers \& Cooper, 1997). Así, estos resultados de alguna manera coinciden con los hallazgos de Gil-Monte \& Peiró (1999), quien encuentra relación significativa entre la satisfacción laboral con tres dimensiones del Inventario de Burnout de Maslach (MBI). Igualmente, coincide con los resultados encontrados por Grajales (2000) en la existencia de una relación negativa significativa entre el agotamiento emocional y las variables Satisfacción Laboral e involucramiento en el trabajo en una muestra de docentes de la Universidad de Nuevo León, en México.

Asimismo, la tercera hipótesis también se confirma, es decir, la satisfacción laboral de los docentes de universidades estatales difiere de los docentes de las universidades privadas de Lima metropolitana. Los docentes de las universidades privadas obtienen puntajes promedios más altos en los factores Intrínseco, Extrínseco y en la escala total que los profesores de universidades estatales, encontrándose diferencias estadísticamente significativas en 
estos. Del mismo modo, se encuentran diferencias estadísticamente significativas entre los docentes de universidades estatales y docentes de universidades privadas correlaciones en los subfactores Independencia, Variedad, Libertad de cátedra, Reconocimiento y distinciones, Compensación económica, Condiciones de trabajo, Estatus, Calidad de relaciones interpersonales y Capacitación y perfeccionamiento. Se encuentran diferencias pero no significativas estadísticamente en el factor Seguridad en el puesto. Los resultados obtenidos pueden explicarse por diferentes factores, como infraestructura, tecnología, organización, remuneraciones, recursos asignados a la investigación, entre otros. De otro lado, la percepción del público de la educación impartida por las universidades privadas como de muy buena calidad, de confianza, y que últimamente ha mejorado (Universidad de Lima, 2004) 1levaría a que los docentes obtengan puntajes más elevados en Satisfacción Laboral. Asimismo, estos hallazgos coinciden con los resultados obtenidos por Fernández (2002) en una muestra de docentes de primaria de Lima metropolitana.

Igualmente, también se valida la tercera hipótesis, es decir, las Fuentes de Presión Laboral de los docentes de universidades estatales difieren de las de los docentes de las universidades privadas de Lima metropolitana. Los profesores de las universidades estata- les obtienen puntajes promedios mayores en las escalas de presiones Derivadas de la labor educativa en el aula, Derivadas de la vida cotidiana en el aula, Derivadas de la organización administrativa y en los factores derivados de la Flexibilidad con respecto a la tarea docente que los profesores de universidades privadas. También se encuentran diferencias significativas entre ambos grupos. Los factores indicados en el tercer objetivo a los que se enfrenta el docente explicarían que quienes laboran en universidades estatales obtienen mayores promedios en el Cuestionario de Fuentes de Presión Laboral. Asimismo, estos resultados coinciden con los resultados encontrados por Fernández (2002) en docentes de educación primaria y Delgado, Escurra y Atalaya (2003) en docentes de educación secundaria.

La tercera hipótesis también se confirma, esto es, los factores de las Fuentes de Presión Laboral influyen sobre los factores de la Satisfacción Laboral. Los resultados del análisis multivariado del modelo de ecuaciones estructurales permite aceptar la hipótesis propuesta. Así, se encuentra que el factor derivado de la Labor educativa en el aula influye negativamente sobre la Satisfacción Extrínseca; el factor derivado de la Vida cotidiana en el aula influye negativamente sobre la Satisfacción Intrínseca; el factor originado por la Organización administrativa influye negativamente sobre la Satisfac- 
ción Extrínseca e Intrínseca; el factor Flexibilidad con respecto a la tarea docente, influye positivamente en la satisfacción Extrínseca e Intrínseca.

Por otra parte, al realizar el análisis comparativo según las variables asignadas encontramos que se presentan algunas diferencias entre los grupos de variables consideradas en la presente investigación. De esta manera, cuando comparamos a los docentes según el ejercicio de la docencia en pregrado y posgrado solo se encuentran diferencias estadísticamente significativas en las escalas Extrínseca y Total. Asimismo, se encuentra que los docentes que ejercen la docencia en pregrado obtienen promedios superiores en todos los subfactores de la escala de satisfacción laboral, con excepción en el subfactor Línea de carrera. Se encuentran diferencias significativas entre ambos grupos en los subfactores Independencia, Variedad, Libertad de cátedra y Calidad de relaciones interpersonales. En relación con las Fuentes de Presión Laboral no se encuentran diferencias significativas entre los docentes de ambos grupos en la referida escala.

Al comparar los resultados obtenidos por los docentes según la condición de nombrado y contratado se encuentran diferencias estadísticamente significativas en los subfactores Reconocimiento y distinciones, Seguridad en el puesto, Calidad de relaciones interpersonales y Capacitación y perfecciona- miento. En la Escala de Presión Laboral no se encuentran diferencias estadísticamente significativas en los cuatro factores ni en la escala total entre ambos grupos. Estos últimos resultados contrastan con los obtenidos en la investigación de Cifre \& Lorens (2002) al analizar las principales diferencias en relación con los estresores laborales, bournout y bienestar psicológico entre los profesores de la Universidad Jaume I de Castellón, atendiendo a la categoría laboral (tiempo parcial, tiempo completo y funcionarios). Los resultados de esta investigación reportan que solo dos de las demandas estudiadas (sobrecarga emocional y sobrecarga cuantitativa) se consideran estresores que permiten diferenciar entre las distintas condiciones de docentes de la referida universidad. Los resultados pueden deberse a que los docentes contratados deben añadir a sus labores un alto nivel de tareas de investigación de alta calidad, que les permita mejorar su currículo para lograr su promoción a funcionario $\mathrm{y}$, por lo tanto, convertirse en personal estable (nombrado) de la universidad.

Al comparar los resultados obtenidos por los docentes según el tiempo de dedicación que asignan a la docencia, encontramos diferencias estadísticamente significativas en la Escala de Satisfacción Laboral en los factores Intrínseco y Extrínseco y en la escala total entre los docentes que trabajan a tiempo completo y los docentes a dedi- 
cación exclusiva, y entre los docentes a tiempo parcial y los de dedicación exclusiva. Asimismo, encontramos diferencias significativas en los subfactores Compensación económica, Condiciones de trabajo, Seguridad en el puesto, Calidad de relaciones interpersonales y Capacitación y perfeccionamiento docente. En la escala de fuentes de presión laboral encontramos diferencias significativas entre los profesores a dedicación exclusiva y a tiempo completo en el factor relacionado con las presiones sociales derivadas de la labor educativa en el aula; asimismo, se encuentran diferencias estadísticamente significativas entre los docentes a dedicación exclusiva y a tiempo completo y entre los docentes con dedicación exclusiva y los que se dedican a tiempo parcial en el factor relacionado con las presiones originadas por la organización administrativa. Este hallazgo contrasta con el de Caramés (2001), quien reporta que no encuentra diferencias entre los profesores de la Universidad de Galicia considerando la jornada laboral.

Con relación a la categoría de los docentes, no se encuentran diferencias significativas entre los docentes principales, asociados y auxiliares en los factores intrínsecos, extrínsecos y en la escala total de satisfacción laboral. Por otro lado, se encuentran diferencias significativas entre los docentes principales y auxiliares en el subfactor Línea de carrera y entre los principales y los auxiliares y entre los asociados y los auxiliares en el subfactor Seguridad en el puesto. En la escala de fuentes de presión no se encuentran diferencias significativas entre los profesores principales y asociados en los factores que se derivan de la labor educativa en el aula, derivados de la vida cotidiana en el aula, en los provenientes de la organización educativa y en el factor flexibilidad con respecto a la tarea docente. Si bien no existen diferencias significativas los docentes principales obtienen promedios superiores que los obtenidos por asociados y auxiliares, explicable por el tiempo que llevan en la docencia y los logros obtenidos a lo largo de su carrera, que también los han llevado a desarrollar mejores estrategias de afrontamiento de estrés y expectativas profesionales más reales.

Los docentes que trabajan en una sola universidad muestran mayores niveles de Satisfacción Laboral que los que trabajan en más de una universidad en los factores Intrínseco, Extrínseco y Total de la Escala de Satisfacción Laboral. Igualmente, manifiestan mayor Satisfacción Laboral en los subfactores Variedad, Libertad de cátedra, Línea de carrera, Reconocimiento y distinciones, Compensación económica, Condiciones de trabajo, Estatus, Calidad de relaciones interpersonales y Capacitación y perfeccionamiento. En la escala de fuentes de presión laboral, como era de esperarse, los docentes que trabajan en más de una universidad muestran 
mayores puntajes promedio que los que trabajan en una universidad en los factores derivados de la labor educativa en el aula, en los derivados de la vida cotidiana en el aula, derivados de la organización administrativa y las relacionadas con la flexibilidad de la tarea docente. Estos resultados coinciden con los hallazgos de Escurra (1999), quien en una investigación orientada a conocer las características psicológicas, consecuencias en el comportamiento, desempeño, estrés y satisfacción laboral de profesionales que trabajan en uno o en varios empleos. Los resultados indican que los profesionales universitarios pluriempleados no están satisfechos con su actividad laboral ni con sus compañeros, explicándose estos hallazgos debidos a la rotación constante que debe realizar en diferentes organizaciones en las cuales laboran y que puede llevarlos a tener un contacto superficial con sus ocasionales compañeros y no llegar a integrarse e identificarse con las instituciones en las que se desempeñan, lo que no ocurre en los casos de profesionales con empleo único. Asimismo, los pluriempleados exhiben una mayor satisfacción en el aspecto económico, que es lo esperado debido a los esfuerzos desplegados para conseguirlo. En cuanto a las fuentes de estrés, los pluriempleados obtienen mayores niveles significativos en el estrés laboral que los uniempleados, posiblemente, debido a la mayor cantidad de situaciones problemáticas que deben enfrentar y que son percibidas como de mayor intensidad.

Los docentes que se formaron académicamente en universidades privadas obtienen mayores puntajes promedio en la Escala de Satisfacción Laboral en los factores Intrínseco, Extrínseco y Total que los docentes cuya formación se realizó en universidades estatales. Asimismo, se encuentran diferencias estadísticamente significativas a favor de los docentes que estudiaron en universidades privadas en todos los subfactores de la Escala de Satisfacción Laboral, excepto en los subfactores Seguridad en el puesto y Estatus. De otra parte, los docentes cuya formación profesional se realizó en universidades estatales muestran puntajes promedio mayores que los formados en universidades privadas en los cuatro factores del inventario de presiones a las que se enfrenta el docente. Esto puede explicarse como la expresión de que los docentes de universidades privadas tienen mayores niveles de competitividad que los formados en universidades estatales. A esto debe agregarse que en la percepción del público las universidades privadas son consideradas como confiables, de buena calidad académica y más estimuladoras de la competitividad.

No se encuentran diferencias estadísticamente significativas en los factores Intrínseco, Extrínseco y Total de la Escala de Satisfacción Laboral entre los 
docentes, según la variable estado civil soltero y casado. Se encuentra mayor satisfacción laboral entre los docentes casados en el subfactor Seguridad en el puesto. A su vez, estos presentan mayores puntajes promedio que los solteros en los factores de presiones Derivadas de las actividades educativas, Derivadas de la vida cotidiana en el aula, Flexibilidad de la tarea docente y puntaje total de la Escala de Fuentes de Presión Laboral. Así, Evans y Bartolomé (1984, citado en Buendía, 1993) señalan cinco tipos de relaciones posibles entre trabajo y familia. En primer lugar, el trabajo y la familia pueden afectarse uno a otro, positiva o negativamente; por ejemplo, la Satisfacción Laboral puede contribuir a la satisfacción de la vida familiar, mientras que la insatisfacción en alguno de los dos aspectos puede contribuir a la insatisfacción en esta. Las relaciones trabajo-familia pueden ser independientes (independencia entre ambas), conflictivas (el éxito en uno supone sacrificio en el otro, instrumentales (el trabajo puede ser una forma de mantener satisfactoria la vida familiar o viceversa) y, por último, pueden compensarse (cuando la familia proporciona los recursos que se pierden en el trabajo).

En lo que respecta al género, no se encuentran diferencias estadísticamente significativas en los factores Intrínseco, Extrínseco y en los diferentes subfactores de la Escala de Satisfacción Laboral. Tampoco se encuentran diferencias estadísticamente significativas en la Escala de Presión Laboral.
Estos resultados coinciden con los obtenidos por Fernández (2001) en Satisfacción laboral y Fuentes de presión laboral en docentes de educación primaria de Lima metropolitana. Asimismo, con los resultados obtenidos por Delgado, Escurra \& Atalaya (2002), donde no se encuentran diferencias en los niveles de estrés laboral entre docentes varones y mujeres de educación secundaria de Lima metropolitana. Asimismo, coincide en que no existen diferencias significativas según género encontradas en otras investigaciones (Grajales, 2000; Caramés, 2001; Speelberg, Reheiser \& Vagg, 1999). De otro lado, contrastan con los hallazgos realizados en otras investigaciones donde se reportan diferencias significativas a nivel de género y estrés laboral (Bosch, 2002; Gil-Monte \& Peiró, 1997, 1999).

Al dividir el grupo de docentes según lugar de procedencia no se encuentran diferencias estadísticamente significativas entre los nacidos en Lima y aquellos nacidos en provincias en los factores Intrínseco, Extrínseco y puntaje total de la Escala de Satisfacción Laboral. Tampoco se encuentran diferencias significativas entre los docentes según lugar de nacimiento en los diferentes factores de la Escala de Fuentes de Presión Laboral.

También se dividió al grupo de docentes según el tiempo de servicios prestados a la universidad y los años de trabajo en la docencia universitaria. 
Considerando los años de trabajo en la docencia se encuentra correlación positiva baja con el factor seguridad en el puesto; en tanto que a los años de trabajo en la universidad donde presta servicios se encuentra correlación negativa baja con los factores Intrínseco, Total, Independencia, Reconocimiento y distinciones, Compensación económica, Condiciones de trabajo, Seguridad en el puesto y Capacitación y perfeccionamiento docente. En la Escala de Fuentes de Presión Laboral no se encuentra correlación entre los factores de la escala y las variables Años de trabajo en la docencia y Años de trabajo en la universidad.

De otro lado, se han identificado tres grupos de docentes según la Satisfacción Laboral y las Fuentes de Presión Laboral: alto, medio y bajo. Los docentes de universidades de gestión estatal que afrontan niveles de Presión Laboral altos manifiestan Satisfacción Laboral media y baja y los que presentan Presión Laboral media y baja muestran baja satisfacción. En tanto que los docentes sometidos a niveles de Presión Laboral alta muestran niveles de Satisfacción Laboral media y alta y los docentes con Presión Laboral media y baja muestran Satisfacción Laboral media y alta.

Se han identificado los factores de la Presión Laboral que tienen mayor y menor influencia en el estrés laboral y el factor y subfactores de las escalas de Satisfacción Laboral del docente uni- versitario de Lima metropolitana. En las Fuentes de Presión Laboral, los factores originados por la organización administrativa tienen el mayor peso como elemento que contribuye al estrés laboral; en tanto que el factor derivado de la labor educativa en el aula tiene menor influencia.

Por otra parte, el factor Intrínseco y los subfactores Independencia, Estatus y Libertad de cátedra tienen mayor incidencia en la Satisfacción Laboral de los docentes universitarios; en tanto que los subfactores Reconocimiento y distinciones, Capacitación y perfeccionamiento y Compensación económica tienen menor incidencia.

Frederick Herzberg propone en su teoría bifactorial sobre la Satisfacción Laboral la existencia de dos factores: intrínsecos o motivadores y extrínsecos o de higiene. Si se satisfacen los factores extrínsecos o higiénicos el trabajador se siente satisfecho y si no se satisfacen se produce la insatisfacción; en tanto que, si se cubren las necesidades intrínsecas o motivadoras el trabajador está satisfecho y si no se cubren estas necesidades se encuentra en un estado neutro o no satisfecho. De acuerdo con los resultados obtenidos podemos afirmar que los subfactores Independencia, Libertad de cátedra y Variedad, que obtienen los mayores promedios, actuarían como factores motivadores. Por otro lado, los subfactores Compensación económica, Capacitación y perfeccionamiento, Seguridad en el puesto 
y Calidad de relaciones interpersonales, que obtienen los menores promedios, actuarían como factores higiénicos que, al no ser cubiertos, serían causantes de la insatisfacción laboral. El subfactor Estatus (extrínseco), que obtiene el segundo lugar en el orden de mérito, desvía la insatisfacción del trabajador hacia una posición neutra, y el subfactor Reconocimiento y distinciones (intrínseco) que no es cubierto lleva al trabajador a un estado de no satisfecho, es decir, un estado neutro.

Finalmente, creemos que es responsabilidad de las autoridades universitarias buscar los mecanismos necesarios, dentro de sus limitaciones, para desviar la insatisfacción de los factores higiénicos en los docentes universitarios hacia una posición neutra y aumentar la satisfacción de los factores intrínsecos para que el docente pase de esta posición a un estado de satisfacción laboral. Igualmente, consideramos como responsabilidad buscar estrategias, individuales, grupales y organizacionales, orientadas a disminuir o atenuar las fuentes de presión laboral.

\section{REFERENCIAS}

Aiken, L. (1996). Tests psicológicos y evaluación. México: Prentice Hall.

Alarcón, R. (1991). Métodos y diseños de investigación del comportamiento. Lima: Universidad Peruana Cayetano Heredia, Fondo Editorial.
Álvarez, D. (1994). Satisfacción laboral en un grupo de trabajadores de una empresa pública. Cuadernos de psicología. Lima: Universidad de Lima. (2), 77-134.

Anastasi, A. \& Urbina, S. (1998). Test psicológicos. México: Prentice Hall.

Arbuckle, J. (2000). Introduction to Structural Equation Modeling using AMOS. Austin: Academic Corporation and Instructional Technology Services.

Arbuckle, J. (2004). AMOS 5.0: Programming reference guide. Chicago: Small Waters Corporation.

Bello, M. (2000). Innovaciones pedagógicas en la educación universitaria peruana. Universidad Peruana Cayetano Heredia, Facultad de Educación. [en línea]. <hppt://www. geocities.com/ Peru/ lecturas/innovaciones.html>.

Bosch, M. (2002). Valores y creencias del profesorado de secundaria sobre algunos aspectos de educación. Revista Electrónica Interuniversitaria de Formación del Profesorado, 5(2) [en línea]. <http://www.3uva.es/ aufop/publica/revelfop.htm>.

Brunner, J. (1993). Algunos criterios para la evaluación de la educación superior y la investigación. Notas para el debate 8. Lima: Grade.

Buendía, J. (Coord.). (1993). Estrés y psicopatología. Madrid: Pirámide. 
Byars, Ll. \& Rue, L. (1996). Gestión de recursos humanos. $4 .^{\text {a }}$ edición. Madrid: Irwin.

Byrne B. M. (2001). Structural Equation Modeling with AMOS: Basic concepts, applications and programming. New Jersey: Lawrence Erlbaum

Campbell, J. J., Dunnette, M. D., Lawler, E. \& Weick, K. E. (1970). Managerial behaviour, Performance and effectiveness. Nueva York: McGraw Hill.

Caramés, R. (2001). Causas del core of bournout del profesorado universitario. VII Congreso Español de Sociología (Salamanca). [en línea] $<$ http://WWW: ase.es $>$.

Cherniss, C. (1992). Long-term consequences of burnout: An exploratory study. Journal of Organizational Behavior, 13(1), 1-11.

Cifre, E. y Llorens, S. (2002). Burnout en profesores de la UJI: Un estudio diferencial. Exposición presentada en el Fòrum de Recerca 7. Universidad Jaume I, Facultad de Ciencias Humanas y Sociales.

Comisión Nacional de la Segunda Reforma Universitaria. (2000). [en línea] $<$ http://www.universia. edu.pe>.

Davis, K. \& Newstrom, J. (1993). El comportamiento humano en el trabajo. México: McGraw-Hill.
Delgado, A., Escurra, M. \& Atalaya, M. (2003). El síndrome del bournout en profesores de educación secundaria de Lima metropolitana. Revista de Investigación en Psicología 6(2) 26-46. Universidad Nacional Mayor de San Marcos.

Escurra, M. (1999). El pluriempleo en profesionales universitarios. Persona 2, 213-249.

Fernández, M. (2002). Satisfacción laboral, fuentes de estrés y bournout en docentes primarios. Tesis para optar el grado de doctor en educación. Universidad Nacional Mayor de San Marcos.

Fernández-Ballesteros, R. (1993). Evaluación conductual hoy. Madrid: Pirámide.

Flórez, J. (1972). El comportamiento humano en las organizaciones. Lima: Universidad del Pacífico.

Galaz Fontes, J. (2002) La satisfacción en el trabajo en académicos de tiempo completo en una universidad estatal. Perfiles educativos XXIV, 96.

García, M. (1993). El estrés en contextos laborales: Efectos psicológicos de los nuevos estresares, en Buendía, J. (compilador). Estrés y Psicopatología. Madrid: Pirámide.

Gil-Monte, P. \& Peiró, J. (1997). Desgaste psíquico en el trabajo: El sindrome de quemarse. Madrid: Editorial Síntesis. 
Gil-Monte, P. \& Peiró, J. (1999). Perspectivas teóricas y modelos interpretativos para el estudio del síndrome de quemarse por el trabajo. Anales de Psicología, 15(2), 261-268.

González, D. (1988). Un modelo estructural de satisfacción docente. Revista mexicana de psicología 15(2), 149-164.

Grajales, T. (200) El agotamiento emocional en los profesores de Nuevo León, México: Un estudio de géneros. [En línea] $<$ http://www.tgrajales.net $>$.

Grupo de Análisis para el Desarrollo (Grade) (1990). Educación superior en el Perú: datos para el análisis. Lima: Grade.

Guerrero, E. (2003). Análisis pormenorizado de los grados de burnout y técnicas de afrontamiento del estrés docente en profesorado universitario. Anales de Psicología 19(1), 145-158.

Guillén, C. \& Gil, R. (2000). Psicología del trabajo para relaciones personales. Madrid: McGraw-Hill/ Interamericana de España.

Hernández, R. \& Fernández, C. \& Baptista, P. (2006). Metodología de la investigación. México: McGrawHill.

Herzberg, F. (1968). ¿Cómo motiva usted a sus empleados? Harvard Business Review 46, 53-62.
Howell, W. (1979). Psicología industrial y organizacional. México: El Manual Moderno.

Instituto Nacional de Estadística e Informática (1996). I Censo Nacional Universitario 1996.

Ivancevich, J. \& Mattesson, M. (1992). Estrés y trabajo. Una perspectiva gerencial. México: Editorial Trillas.

Kerlinger, F. (1991). Investigación del comportamiento. 3. a edición. México: McGraw-Hill.

León, F. (1976). Factores considerados por civiles y militares al "ofrecérseles" un puesto público. Lima: Esan.

Marchand, C. \& Pérez de Maldonado, I. (1997). Clima organizacional y satisfacción en el trabajo en un instituto universitario. Revista Interamericana de Psicología Ocupacional 16(1), 22-29.

Marín, C. (1986). Consideraciones metodológicas para conducir investigaciones psicológicas en América Latina. Acta Psiquiátrica y Psicológica de América Latina 32, 183-192.

Martínez Arias, R. (1985). Psicometría: Teoría de los test psicológicos y educativos. Madrid: Síntesis.

Martínez, J. (2004). Estrés laboral. Guía para empresarios y empleados. Madrid: Prentice Hall. 
Mondelo, P., Gregori, E. \& Barrau, P. (2000). Ergonomía 1. Fundamentos. México: Alfaomega Grupo Editor, S.A. de C.V.

Muchinsky, P. (2002). Psicología aplicada al trabajo: Una introducción a la psicología organizacional. México: Thomson Learning.

Muñiz, J. (1996). Psicometría. Madrid: Universitas.

Muñiz, J. (1994). Teoría clásica de los test. Madrid: Pirámide.

Oficina Internacional del Trabajo. Empleo y condiciones de trabajo del personal docente. Ginebra: OIT.

Padua, J. (1979). Técnicas de investigación aplicadas a las ciencias sociales. México: Fondo de Cultura Económica.

Palma, S. (1999). Elaboración y validación de una Escala de Satisfacción Laboral SL-SPC para trabajadores de Lima metropolitana. Teoría e investigación en Psicología. Lima: Universidad Ricardo Palma $I X(1)$, 27-34.

Palma, S. (2000). Motivación y clima laboral en personal de entidades universitarias. Revista de Investigación en Psicología 3(1), 11-21.

Peiró, J. (1984). Psicología de las organizaciones. Madrid: UNED.

Peiró, J. (1987). Psicología de la organización. Madrid: Universidad Nacional de Educación a Distancia.
Peiró, J. (1996). Tratado de psicología del trabajo. Madrid: Síntesis.

Peiró, J. (2005). Desencadenantes del estrés laboral. Madrid: Pirámide.

Pérez, I. (1995). Satisfacción laboral y comportamientos organizacionales. Revista Interamericana de Psicología Ocupacional 14(1), 7-24.

Pérez, I. (1996). La satisfacción en el trabajo: Un enfoque para su estudio. Revista Interamericana de Psicología Ocupacional 15(2), 65-79.

Polanco R. (1994). Medición y cambio de actitudes en profesores de educación superior. Revista Intercontinental de Psicología 7(1),161-187.

Reimel, S. (1994). Calidad de vida percibida de profesores universitarios: Dos muestras venezolanas. Revista Interamericana de Salud Ocupacional 13(1), 7-19.

Reimel, S. (1998). ¿Para qué se estudia la calidad de vida laboral? Revista Interamericana de Psicología Ocupacional 17(1), 34-53.

Robbins, S. (2004). Comportamiento organizacional $10 .^{\mathrm{a}}$ edición. México: Pearson/Prentice Hall.

Salmi, J. (2000). Educación superior. Enfrentando los retos del siglo XXI. [en línea] <http://www.wordbank. org/education/tertiary>. 
Sánchez, H. \& Reyes, C. (2002). Metodología y diseños en la investigación científica. Lima: Editorial Mantaro.

Sota, J. (2000). El financiamiento de la universidad peruana: Problemas y propuestas. Consorcio de Universidades. La Universidad que el Perú necesita. Lima: Foro Educativo.

Spielberger, C. D., Reheiser, E. \& Vagg, P. (1999). Medición del estrés en el trabajo: La encuesta de estrés laboral. Revista de la Facultad de Psicología de la Universidad Nacional Mayor de San Marcos III(4), 51-68.

Travers, Ch. \& Cooper, C. (1997). El estrés de los profesores. La presión en la actividad docente. Barcelona: Paidós.

Unesco (1995). Política para el cambio de la educación superior.
Unesco (1997). Recomendación relativa a la condición del personal docente de la enseñanza superior.

Unesco (1998). Declaración mundial sobre la educación superior en el siglo XXI: Visión y acción.

Universidad de Lima. (2004). Grupo de Opinión Pública. Estudio 218. Barómetro social. Lima metropolitana y el Callao. Lima, 20-21 de marzo.

U. S. Department of Education, National Center for Education Statistics, National Study of Postsecondary Faculty: 1999. (2002). Faculty satisfaction. NEA Higher Educational. 8,2, mayo (2001). Part-time Faculty. NEA Higher Educational. 7,4, septiembre (2000). The American Faculty Poll. NEA Higher Educational. 6,3, junio.

Weinert, A. (1985). Manual de psicología de la organización. Barcelona: Herder. 
Álvarez 